\title{
Microglial Depletion with CSF1R Inhibitor During Chronic Phase of Experimental Traumatic Brain Injury Reduces Neurodegeneration and Neurological Deficits
}

\author{
Rebecca J. Henry, ${ }^{1}$ Rodney M. Ritzel, ${ }^{1}$ James P. Barrett, ${ }^{1}$ Sarah J. Doran, ${ }^{1}$ Yun Jiao, ${ }^{2}$ Jennie B. Leach, ${ }^{2}$ \\ ๑Gregory L. Szeto, ${ }^{2,3,4}$ Junfang Wu, ${ }^{1}{ }^{\odot B}$ Bogdan A. Stoica, ${ }^{1,5,6}$ Alan I. Faden, ${ }^{1}$ and $\odot$ David J. Loane ${ }^{1,7}$ \\ ${ }^{1}$ Department of Anesthesiology and Shock, Trauma and Anesthesiology Research (STAR) Center, University of Maryland School of Medicine, Baltimore, \\ Maryland 21201, ${ }^{2}$ Department of Chemical, Biochemical and Environmental Engineering, University of Maryland, Baltimore County, Maryland 21250, \\ ${ }^{3}$ Marlene and Stewart Greenebaum Comprehensive Cancer Center, University of Maryland, Baltimore, Maryland 21201, ${ }^{4}$ Department of Microbiology and \\ Immunology, University of Maryland School of Medicine, Baltimore, Maryland 21201, 5Division of Translational Radiation Sciences (DTRS), Department of \\ Radiation Oncology, University of Maryland School of Medicine, Baltimore, Maryland 21201, ${ }^{6}$ VA Maryland Health Care System, Baltimore VA Medical \\ Center, Baltimore Maryland 21201, and 7 School of Biochemistry and Immunology, Trinity Biomedical Sciences Institute, Trinity College, Dublin, Ireland
}

Chronic neuroinflammation with sustained microglial activation occurs following severe traumatic brain injury (TBI) and is believed to contribute to subsequent neurodegeneration and neurological deficits. Microglia, the primary innate immune cells in brain, are dependent on colony stimulating factor 1 receptor (CSF1R) signaling for their survival. In this preclinical study, we examined the effects of delayed depletion of chronically activated microglia on functional recovery and neurodegeneration up to 3 months postinjury. A CSF1R inhibitor, Plexxikon (PLX) 5622, was administered to adult male C57BL/6J mice at 1 month after controlled cortical impact to remove chronically activated microglia, and the inhibitor was withdrawn 1-week later to allow for microglial repopulation. Following TBI, the repopulated microglia displayed a ramified morphology similar to that of Sham uninjured mice, whereas microglia in vehicle-treated TBI mice showed the typical chronic posttraumatic hypertrophic morphology. PLX5622 treatment limited TBI-associated neuropathological changes at 3 months postinjury; these included a smaller cortical lesion, reduced hippocampal neuron cell death, and decreased NOX2and NLRP3 inflammasome-associated neuroinflammation. Furthermore, delayed depletion of chronically activated microglia after TBI led to widespread changes in the cortical transcriptome and altered gene pathways involved in neuroinflammation, oxidative stress, and neuroplasticity. Using a variety of complementary neurobehavioral tests, PLX5622-treated TBI mice also had improved long-term motor and cognitive function recovery through 3 months postinjury. Together, these studies demonstrate that chronic phase removal of neurotoxic microglia after TBI using CSF1R inhibitors markedly reduce chronic neuroinflammation and associated neurodegeneration, as well as related motor and cognitive deficits.

Key words: CSF1R; functional recovery; microglia; neurodegeneration; neuroinflammation; traumatic brain injury

Significance Statement

Traumatic brain injury (TBI) is a debilitating neurological disorder that can seriously impact the patient's quality of life. Microglial-mediated neuroinflammation is induced after severe TBI and contributes to neurological deficits and on-going neurodegenerative processes. Here, we investigated the effect of breaking the neurotoxic neuroinflammatory loop at 1-month after controlled cortical impact in mice by pharmacological removal of chronically activated microglia using a colony stimulating factor 1 receptor (CSF1R) inhibitor, Plexxikon 5622. Overall, we show that short-term elimination of microglia during the chronic phase of TBI followed by repopulation results in long-term improvements in neurological function, suppression of neuroinflammatory and oxidative stress pathways, and a reduction in persistent neurodegenerative processes. These studies are clinically relevant and support new concepts that the therapeutic window for TBI may be far longer than traditionally believed if chronic and evolving microglial-mediated neuroinflammation can be inhibited or regulated in a precise manner. 


\section{Introduction}

Traumatic brain injury (TBI) is a major cause of morbidity and mortality world-wide (Maas et al., 2017). Its high incidence and prevalence result in a large number of survivors with significant long-term disabilities (Bryant et al., 2010; Sabaz et al., 2014; Kumar et al., 2018). Traumatic insults to the brain produce tissue damage and related neurological dysfunction through both direct mechanical damage (primary injury) and more delayed mechanisms that result from secondary biochemical/metabolic alterations (Loane and Faden, 2010). Recent clinical and experimental TBI studies have implicated chronic neuroinflammation as an important secondary injury mechanism that may be treatable (Simon et al., 2017).

Following brain trauma, microglia become activated and undergo phenotypic and functional changes (Kumar et al., 2016a). Microglial activation is an essential innate immune response that is required to protect the brain from CNS injury (Salter and Stevens, 2017). However, uncontrolled activation, or activation over extended periods, is detrimental and can contribute to neurodegenerative processes and related neurobehavioral dysfunction (Block et al., 2007). Chronic microglial activation has been reported in TBI patients for many years after the initial brain trauma (Gentleman et al., 2004; Ramlackhansingh et al., 2011; Johnson et al., 2013; Coughlin et al., 2015, 2017; Cherry et al., 2016), and is more broadly distributed than suggested by the primary lesion. Moreover, it is involved in persistent white matter atrophy and related cognitive decline (Maxwell et al., 2006; Bendlin et al., 2008; Ramlackhansingh et al., 2011; Johnson et al., 2013; Coughlin et al., 2015; Wilson et al., 2017). Experimental studies implicate NADPH oxidase (NOX2) as a common and necessary mechanism of microglia-mediated neurotoxicity during chronic neurodegeneration (Babior, 1999; Qin et al., 2004; Block et al., 2007). Additionally, we reported that inhibiting NOX2 activity after TBI in mice suppressed microglial neurotoxicity, resulting in reduced tissue loss and improved long-term functional recovery (Kumar et al., 2016a,b; Barrett et al., 2017).

Microglia express colony stimulating factor 1 receptor (CSF1R) (Erblich et al., 2011; Nandi et al., 2012), and CSF1R signaling is essential for microglial development and their longterm survival (Ginhoux et al., 2010; Erblich et al., 2011). The recent discovery of CSF1R small-molecule inhibitors has enabled selective elimination of microglia under both physiological (Elmore et al., 2014) and pathological conditions (Szalay et al., 2016; Jin et al., 2017; Li et al., 2017). Oral administration of CSF1R inhibitors rapidly deplete $>95 \%$ of all microglia in the CNS within $7 \mathrm{~d}$ of treatment (Elmore et al., 2014). Notably, in a mouse hippocampal lesion model, depletion of microglia by a $30 \mathrm{~d}$ treatment with a CSF1R inhibitor, Plexxikon (PLX) 3397, improved

Received 0ct. 7, 2019; revised Jan. 3, 2020; accepted Jan. 23, 2020.

Author contributions: R.J.H., B.A.S., and D.J.L. designed research; R.J.H., R.M.R., J.P.B., and S.J.D. performed research; R.J.H., R.M.R., J.P.B., S.J.D., Y.J., J.B.L., G.L.S., B.A.S., and D.J.L. analyzed data; R.J.H. wrote the first draft of the paper; G.L.S., A.I.F., and D.J.L. edited the paper; J.W. contributed unpublished reagents/analytic tools; D.J.L. wrote the paper.

This work was supported by the National Institutes of Health (Grant R01NS082308 to D.J.L., Grant R01NS037313 to A.I.F., Grant R01NS096002 to B.A.S., Grant R01NS110756 to D.J.L., A.I.F., and B.A.S., and Grant R21EY029451 to J.B.L.), the U.S. Department of Veterans Affairs (Grant 1101 RX001993 to B.A.S.), and Science Foundation Ireland (Grant 17/FRL/4860 to D.J.L.). We thank Victoria Meadows and Wesley Shoap for help with neurobehavioral assessment and histology and Plexxikon Inc. for the use of PLX5622.

The authors declare no competing financial interests.

Correspondence should be addressed to Rebecca J. Henry at rhenry@som.umaryland.edu or David J. Loane at loanedj@tcd.ie.

https://doi.org/10.1523/JNEUROSCI.2402-19.2020

Copyright $\odot 2020$ the authors cognitive recovery and reduced proinflammatory gene expression in the injured hippocampus (Rice et al., 2015). Despite evidence that chronic and evolving microglial activation is detrimental following severe TBI (Byrnes et al., 2012; Loane et al., 2014; Pischiutta et al., 2018), prolonged inhibition of microglia is unlikely to be therapeutic because microglia perform critical functions such as synaptic pruning and restoration of tissue homeostasis after pathological insult (Salter and Stevens, 2017). However, the withdrawal of CSF1R inhibitors in microgliadepleted mice results in rapid self-renewal and repopulation of microglia in the CNS (Elmore et al., 2014, 2015), which can improve cognitive function recovery in hippocampal lesion studies (Rice et al., 2017). Therefore, removing neurotoxic microglia by short-term treatment with CSF1R inhibitors may have a longterm effect on the lesion microenvironment and disrupt the chronic trajectory of neurodegeneration.

Here, we investigated whether short-term elimination of microglia during the chronic phase of TBI followed by repopulation can reduce posttraumatic neurodegeneration and limit longterm neurological dysfunction. Our findings strongly implicate chronic neurotoxic microglial activation as a major pathophysiological factor in posttraumatic neurodegeneration and related neurological impairments after brain trauma.

\section{Materials and Methods}

\section{Animals}

Studies were performed using adult male C57BL/6J mice (10-12 weeks old; Taconic). Mice were housed in the animal care facility at the University of Maryland School of Medicine under a $12 \mathrm{~h}$ light/dark cycle, with ad libitum access to food and water. All surgical procedures were performed in accordance with protocols approved by the Institutional Animal Care and Use Committee at the University of Maryland School of Medicine.

\section{Experimental design}

C57BL/6 mice ( $n=15-20 /$ group) were subjected to either controlled cortical injury (CCI) or Sham surgery. At $28 \mathrm{~d}$ postinjury (DPI), animals were placed on PLX5622 (1200 ppm; Plexxikon) or vehicle (Veh) chow for 1 week to deplete microglia (Fig. 1A). Animals were returned to a standard chow diet to allow for microglial repopulation, and were maintained on standard chow for the remainder of the study. Following completion of comprehensive behavioral testing at 84 DPI, mice were anesthetized $(100 \mathrm{mg} / \mathrm{kg}$ sodium pentobarbital, I.P.) and transcardially perfused with ice-cold $0.9 \%$ saline $(100 \mathrm{ml})$, followed by $300 \mathrm{ml}$ of $4 \%$ paraformaldehyde (PFA). Brains were removed and postfixed in $4 \%$ PFA overnight, cryoprotected in 30\% sucrose, and were processed for histological outcome measures. A separate cohort of mice ( $n=8-9$ /group) underwent either CCI or Sham surgery. At 28 DPI, animals were placed on PLX5622 or control chow as described above. At 56 DPI, mice were anesthetized $(100 \mathrm{mg} / \mathrm{kg}$ sodium pentobarbital, I.P.) and transcardially perfused with ice-cold $0.9 \%$ saline $(100 \mathrm{ml})$, the ipsilateral brain tissue was rapidly dissected and processed for flow cytometry, or RNA/Nanostring analysis. We chose to deplete microglia at 28 DPI because previous preclinical studies demonstrated the effectiveness of therapeutically targeting chronic neuroinflammatory responses at 1 month after experimental TBI (Byrnes et al., 2012; Piao et al., 2013).

\section{CCI}

Our custom-designed CCI injury device consists of a microprocessorcontrolled pneumatic impactor with a $3.5 \mathrm{~mm}$ diameter tip. Briefly, mice were anesthetized with isoflurane evaporated in a gas mixture containing $70 \% \mathrm{~N}_{2} \mathrm{O}$ and $30 \% \mathrm{O}_{2}$ and administered through a nose mask (induction at $4 \%$ and maintenance at $2 \%$ ). A $10 \mathrm{~mm}$ midline incision was made over the skull, the skin and fascia were reflected, and a $4 \mathrm{~mm}$ craniotomy was made on the central aspect of the left parietal bone. The impounder tip of the injury device was then extended to its full stroke distance (44 mm), positioned to the surface of the exposed dura, and reset to impact the 
cortical surface. Moderate-level CCI was induced using an impactor velocity of $6 \mathrm{~m} / \mathrm{s}$ and deformation depth of $2 \mathrm{~mm}$ (Loane et al., 2014; Kumar et al., 2016a; Henry et al., 2019). Sham animals underwent the same procedure as TBI mice except for the craniotomy and impact.

\section{PLX5622 administration}

PLX5622 was provided by Plexxikon and formulated in AIN-76A rodent chow by Research Diets at a concentration of $1200 \mathrm{ppm}$ (Spangenberg et al., 2019). Standard AIN-76A diet was provided as Veh control. Mice were provided ad libitum access to PLX5622 or Veh diet for $7 \mathrm{~d}$ to deplete microglia. This dose and time resulted in depletion of $95 \%$ of microglia (Fig. 1C)

\section{Neurobehavioral testing}

Beam walk $(B W)$. Fine motor coordination was assessed using a BW test, as described previously (Kumar et al., 2016b; Henry et al., 2019). Briefly, mice were placed on one end of a wooden beam $(5 \mathrm{~mm}$ wide and $120 \mathrm{~mm}$ in length), and the number of foot faults (ff) of the right hindlimb were recorded over 50 steps. Mice were trained on the BW for $3 \mathrm{~d}$ before Sham or CCI and tested weekly through 84 DPI.

Accelerating rotorod. Gross motor function and balance was assessed on the accelerating Rotorod at 84 DPI, as described previously (Doran et al., 2019). On the day of testing, mice were placed on the rod which accelerated from 4 to 60 RPM with maximal speed reached within $20 \mathrm{~s}$. Latency to fall from the rod (or cling to and rotate with the rod for three consecutive rotations) was recorded for three trials. A 5-10 min rest period with access to food and water was allowed between each trial. Scores from the three trials were averaged to give a single score for each mouse.

$Y$-maze maze. The Y-maze was performed at 70 DPI to test spatial working memory in mice, as described previously (Kumar et al., 2016b; Henry et al., 2019). The Y-maze (Stoelting, Wood Dale, IL) consisted of three identical arms, each arm $35 \mathrm{~cm}$ long, $5 \mathrm{~cm}$ wide, and 10 $\mathrm{cm}$ high, at an angle of $120^{\circ}$ with respect to the other arms. One arm was randomly selected as the "start" arm, and the mouse was placed within and allowed to explore the maze freely for $5 \mathrm{~min}$. Arm entries (arms A-C) were recorded by analyzing mouse activity using ANY-maze software (Stoelting). An arm entry was attributed when all four paws of the mouse entered the arm, and an alternation was designated when the mouse entered three different arms consecutively. The percentage of alternation was calculated as follows: total alternations $\times$ $100 /$ (total arm entries -2 ). If a mouse scored significantly greater than $50 \%$ alternations (the chance level for choosing the unfamiliar arm), this was indicative of spatial working memory.

Novel object recognition (NOR). NOR was performed on 77-78 DPI to assess nonspatial hippocampal-mediated memory, as described previously (Zhao et al., 2012). Mice were placed in an open field $(22.5 \mathrm{~cm} \times$ $22.5 \mathrm{~cm}$ ) and two identical objects were placed near the left and right corners of the open field for training (familiar phase). Mice were allowed to freely explore until they spent a total of $20 \mathrm{~s}$ exploring the objects (exploration was recorded when the front paws or nose contacted the
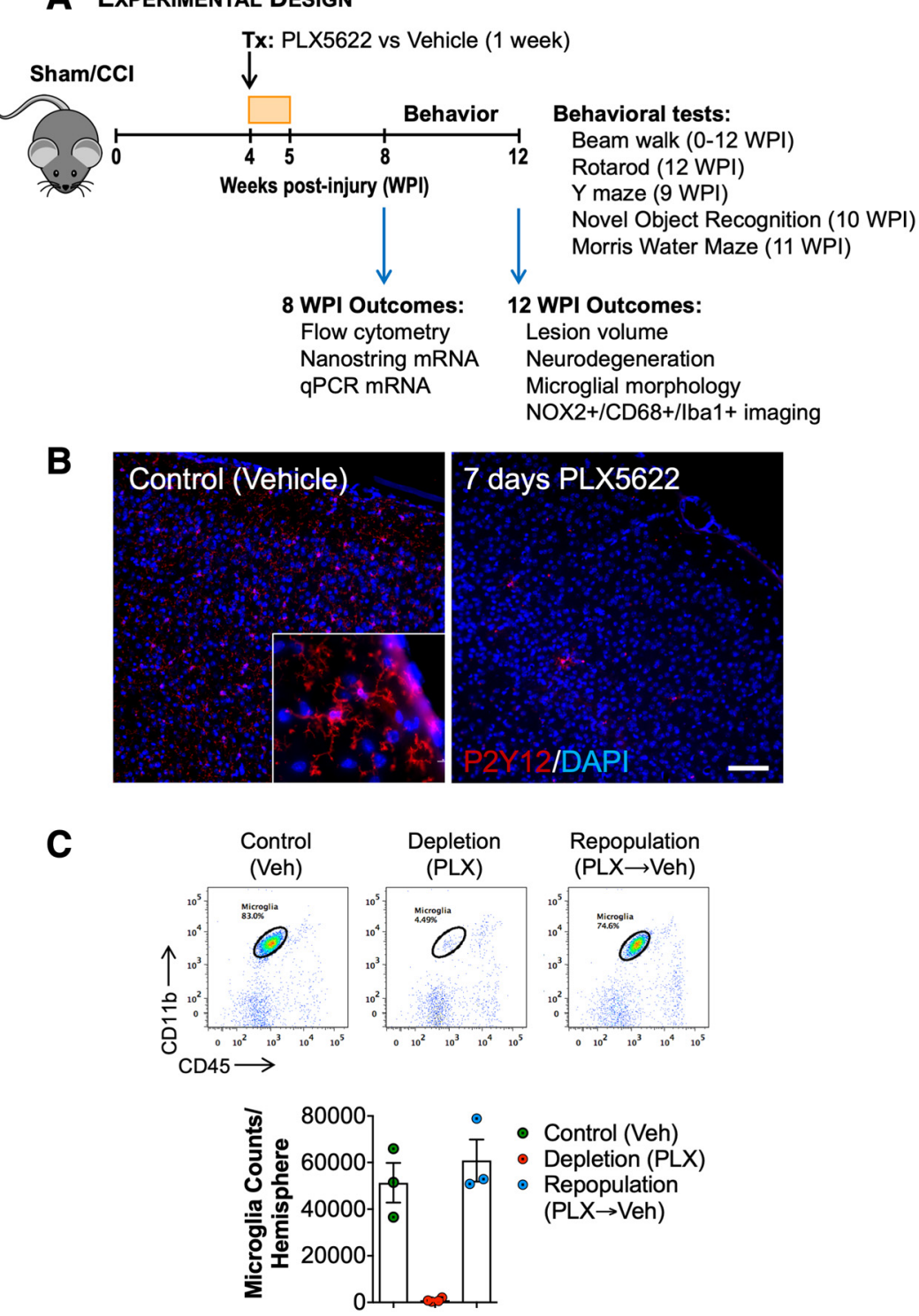

Figure 1. Experimental timeline and in vivo characterization of the CSF1R inhibitor, PLX5622. A, Experimental timeline: adult male C57BL/6 mice underwent CCI/Sham surgery. At 4 weeks after CCI/Sham surgery mice were placed on PLX5622 (1200 ppm) or normal chow (Veh) for 1 week, following which mice were returned to normal chow for the remainder of the study. One cohort of mice were killed at $8 \mathrm{WPI}$ and samples were collected for flow cytometry and nanostring analysis. A separate cohort of mice underwent a battery of neurobehavioral tasks (BW, Y maze, NOR, MWM) through 12 WPI, and samples were collected for histological analysis (Stereology). B, Representative immunofluorescence images of P2Y12 ${ }^{+}$microglia demonstrated that oral administration of PLX5622 (1200 ppm) in chow for $7 \mathrm{~d}$ leads to an almost complete depletion of microglia (P2Y12 ${ }^{+}$, red) in the mouse brain. Scale bar, $200 \mu \mathrm{m}$. C, Flow cytometry analysis of microglia (CD11b ${ }^{+} /$CD $45^{\text {int }}$ ) demonstrated that 7-day PLX5622 treatment resulted in $\sim 95 \%$ depletion of microglia counts in the mouse brain. Returning animals to normal chow feed resulted in repopulation of microglia in mouse brain within $7 \mathrm{~d}$ of removing PLX5622 chow. Data expressed as mean \pm SEM ( $n=3 /$ group).

object). The time spent with each object was recorded using Any-Maze software (Stoelting). After $24 \mathrm{~h}$, object recognition was tested by substituting a novel object for a familiar training object (the novel object location was counterbalanced across mice). Because mice inherently prefer to explore novel objects, a preference for the novel object (more time than chance $[15 \mathrm{~s}]$ spent with the novel object) indicates intact memory for the familiar object.

Morris water maze (MWM). Spatial learning and memory was assessed using the MWM, as described previously (Byrnes et al., 2012; Zhao et al., 2012). The MWM protocol included two phases: (1) hidden platform training (learning acquisition) and (2) a twenty-four-hour probe test (reference memory). A circular tank ( $100 \mathrm{~cm}$ in diameter) was filled with water $\left(23 \pm 2^{\circ} \mathrm{C}\right)$ and was surrounded by various extra-maze cues on the 
wall of the testing area. A transparent platform $(10 \mathrm{~cm}$ in diameter) was submerged $0.5 \mathrm{~cm}$ below the surface of the water. Starting at 79 DPI, the mice were trained to find the hidden submerged platform located in the northeast (NE) quadrant of tank for 4 consecutive days (79-83 DPI). The mice underwent four trials per day, starting from a randomly selected release point (east, south, west, and north). Each mouse was allowed a maximum of $90 \mathrm{~s}$ to find the hidden submerged platform. The latency to find the submerged platform was recorded using Any-Maze software (Stoelting). Reference memory was assessed by a probe test performed $24 \mathrm{~h}$ after the final acquisition day. The platform was removed and the mice were released from the southwest position, and the time in the target quadrant was recorded. Search strategy analysis was performed, as described previously (Byrnes et al., 2012; Zhao et al., 2012). Three strategies were identified using the following categorization scheme: (1) a spatial search strategy was defined as swimming directly to platform with no more than one loop or swimming directly to the correct target quadrant and searching for the platform; (2) a nonspatial systematic strategy was defined as searching the interior portion of or the entire tank without spatial bias, including searching within the incorrect target quadrant before finding platform; and (3) a repetitive looping strategy was defined as circular swimming around the tank, swimming in tight circles, or swimming around the wall of tank before finding the submerged hidden platform. The search strategies were analyzed during the MWM probe test and the percentage of each strategy in each group was calculated using a $\chi^{2}$ analysis. Mice that remained immobile throughout the $90 \mathrm{~s}$ trial in the MWM test were excluded from the analysis (Sham: $n=2$; TBI+Veh: $n=3$; TBI+PLX: $n=2$ ).

\section{Histology}

Lesion volume. Lesion volume was measured on $60 \mu \mathrm{m}$ coronal sections that were stained with cresyl violet (FD NeuroTechnologies; $n=8-12$ / group). Quantification was based on the Cavalieri method using Stereoinvestigator software (MBF Biosciences), as described previously (Byrnes et al., 2012; Henry et al., 2019). The lesion volume was quantified by outlining the missing tissue on the injured hemisphere using the Cavalieri estimator with a grid spacing of $0.1 \mathrm{~mm}$. Every eighth section from a total of 96 sections was analyzed beginning from a random start point.

Neuronal loss. For analysis of posttraumatic neuronal cell loss $60 \mu \mathrm{m}$ coronal sections were stained with cresyl violet, and the optical fractionator method of stereology using Stereoinvestigator software was used. Neurons in the ipsilateral cortex were characterized according to previously described methods (García-Cabezas et al., 2016). For neuronal loss in cortex and dentate gyrus (DG) region of the hippocampus, every fourth $60 \mu \mathrm{m}$ section between -1.34 and $-2.54 \mathrm{~mm}$ and -2.70 to -3.16 $\mathrm{mm}$, respectively, from bregma was analyzed beginning from a random start point. A total of 6 sections were analyzed. The number of surviving neurons in each field was divided by the volume of the region of interest to obtain the cellular density expressed in counts $/ \mathrm{mm}^{3}$.

Microglia morphological analysis. Immunohistochemistry was performed on $60 \mu \mathrm{m}$ coronal sections to assess morphological activation states of microglia in the perilesional cortex, as described previously (Byrnes et al., 2012; Loane et al., 2014). Sections were incubated overnight with rabbit anti-Iba-1 (1:5000; Wako Chemicals), washed in $1 \times$ PBS (three times), and incubated with biotinylated anti-rabbit IgG antibody (Vector Laboratories) for $2 \mathrm{~h}$ at room temperature. Sections were incubated in avidin-biotin-horseradish peroxidase solution (Vectastain Elite ABC kit; Vector Laboratories) for $1 \mathrm{~h}$ and then reacted with 3,3'diaminobenzidine (Vector Laboratories) for color development. Iba-1stained sections were counterstained with cresyl violet and mounted for immunohistochemical analysis using a Leica DM4000B microscope. Stereoinvestigator software was used to count and classify the number of cortical microglia for microglial morphologic phenotypes (ramified and hypertrophic/bushy) at $84 \mathrm{DPI}$, using the optical fractionator method ( $n=10-11$ /group).

Immunofluorescence imaging. Immunofluorescence imaging was performed on $20 \mu \mathrm{m}$ coronal brain sections at $\sim-1.70 \mathrm{~mm}$ from bregma. Standard immunostaining techniques were used, as described previously (Henry et al., 2019). Briefly, $20 \mu \mathrm{m}$ brain sections were washed three times with $1 \times$ PBS, blocked for $1 \mathrm{~h}$ in goat serum containing $0.4 \%$ Triton
$\mathrm{X}-100$, and incubated overnight at $4^{\circ} \mathrm{C}$ with a combination of primary antibodies, including mouse anti-gp91 ${ }^{\text {phox }}$ (NOX2; 1:1000; BD Biosciences), rat anti-CD68 (1:1000; AbD Serotec), rabbit anti-Iba1 (1:1000; Wako Chemicals), rabbit anti-P2Y12 (1:1000; AnaSpec), and chicken anti-GFAP (1:500; Abcam). Sections were washed with $1 \times$ PBS (three times), and incubated with appropriate Alexa Fluor-conjugated secondary antibodies (Life Technologies) for $2 \mathrm{~h}$ at room temperature. Sections were washed with $1 \times \mathrm{PBS}$ (three times), counterstained with $4^{\prime}$, 6-diamidino-2-phenylindole (DAPI; $1 \mu \mathrm{g} / \mathrm{ml}$; Sigma-Aldrich), and mounted with glass coverslips using Hydromount solution (National Diagnostics). Images were acquired using a fluorescent Nikon Ti-E inverted microscope at $10 \times($ Plan Apo $10 \times$ NA 0.45$)$ or $20 \times($ Plan APO $20 \times$ NA 0.75) magnification. Exposure times were kept constant for all sections in each experiment. All images were quantified using Nikon ND-Elements Software (AR 4.20.01). Colocalization of NOX2 and CD68 in microglia/macrophage $\left(\mathrm{Ibal}^{+}\right)$was performed by binary operation intersection followed by thresholding. 12 positive regions of interest near the lesion site per mouse were quantified (with $n=5$ mice/group), and was expressed as NOX2 ${ }^{+} \mathrm{CD}_{68}{ }^{+}$cells $/ \mathrm{mm}^{2}$. For GFAP quantification, tiled images of each brain section were at $20 \times$ magnification $(n=5-8$ mice/group). The background was subtracted using a specific reference region just outside each brain section. The area of analysis was the ipsilateral perilesional region (proximal to the lesion site) and the corresponding site in Sham animals. A common threshold was selected for all images to identify the specific cellular GFAP immunostaining. Astrocyte $\mathrm{GFAP}^{+}$expression was quantified and presented as ratio of area of $\mathrm{GFAP}^{+}$immunostaining in the perilesional region $\left(\mu \mathrm{m}^{2}\right)$ or $\mathrm{GFAP}^{+}$ intensity (arbitrary units) in the perilesional region $\left(\mu \mathrm{m}^{2}\right)$.

\section{Molecular and cellular analysis}

Nanostring analysis. RNA was extracted from a frozen tissue punch from the perilesion cortical region using an RNA Plus Universal Mini Kit (Qiagen). Before analysis, RNA quality was determined by Bioanalyzer and all samples had an RNA integrity number (RIN) $>7.5$. RNA. Total RNA (125 ng) was multiplexed and run on an nCounter Mouse Neuropathology panel (Nanostring Technologies) that profiled 770 genes across six fundamental themes in neurodegeneration, including neurotransmission, neuron-glia interaction, neuroplasticity, cell structure integrity, neuroinflammation, and metabolism.

Partial least-squares discriminant analysis (PLSDA) was performed with mixOmics v6.8.2 (Rohart et al., 2017) using normalized gene counts. All discriminant models were evaluated based on ROC curve using 100 iteration of cross-validation and bootstrap resampling with ROC632 v0.6 (Foucher and Danger, 2012). Pairwise differential expression analyses were performed with NanoStringDiff (v3.6.0) (Wang et al., 2016) using raw gene counts along with positive and negative controls and housekeeping genes from NanoString nCounter. Four comparisons were performed: (1) Sham+Veh versus Sham+PLX, (2) Sham+Veh versus $\mathrm{TBI}+\mathrm{Veh}$, (3) $\mathrm{TBI}+\mathrm{Veh}$ versus $\mathrm{TBI}+\mathrm{PLX}$, and (4) TBI+PLX veresus Sham + PLX. Differentially expressed genes were defined as those with an adjusted $p$-value $<0.1$ for either comparison 1 or 3 . Results were plotted using pheatmap v1.0.12 with hierarchical clustering of genes and experimental groups for all differentially expressed genes. Gene expression was normalized across groups as $z$-scores. Heat maps were also independently plotted and clustered by genes only on annotated subsets with defined functional classes. MixOmics, ROC632, NanoStringDiff, and pheatmap packages were all used in Rstudio v3.6.0. Violin plots of normalized gene counts were generated using Prism v8.0.2 (GraphPad), and annotated by median and upper/lower quartiles. Corresponding negative $\log 10$ adjusted $p$-values were plotted for the pairwise comparisons shown as calculated by NanoStringDiff.

Real-time PCR. Quantitative gene expression analysis in the perilesional cortex was performed using Taqman technology, as described previously (Barrett et al., 2017; Henry et al., 2019). Target mRNAs included TaqMan gene expression assays for $\mathrm{Cybb}$ (NOX2) Mm01287743_m1; Cyba (p22 $\left.{ }^{\text {phox }}\right)$ Mm00514478_m1; Ncf1 (p47 $\left.{ }^{\text {phox }}\right)$ Mm00447921_m1; Ncf4 (p40 $\left.{ }^{\text {phox }}\right)$ Mm00476300_m1; Il1b (IL-1 $\beta$ ) Mm01336189_m1; Nlrp3 (NLRP3) Mm00840904_m1; Casp1 (Caspase-1) Mm00438023_m1; Gfap (GFAP) Mm01253033_m1; Gapdh 
(GAPDH) Mm99999915_g1; Applied Biosystems), and analysis was performed on ABI 7900 HT FAST Real Time PCR machine (Applied Biosystems). Samples were assayed in duplicate in one run ( 40 cycles), which was composed of 3 stages: $50^{\circ} \mathrm{C}$ for $2 \mathrm{~min}, 95^{\circ} \mathrm{C}$ for $10 \mathrm{~s}$ for each cycle (denaturation), and a transcription step at $60^{\circ} \mathrm{C}$ for $1 \mathrm{~min}$. Gene expression was normalized by GAPDH and compared with the control sample to determine relative expression values by $2^{-\Delta \Delta C t}$ method.

Flow cytometry. The ipsilateral hemisphere of each mouse was placed in complete Roswell Park Memorial Institute (RPMI) 1640 (Lonza Group) medium. Brain tissue was mechanically digested using a razor blade to mince tissue and was passed through a $70 \mu \mathrm{m}$-filter using RPMI. CNS tissue was then enzymatically digested using DNase $(10 \mathrm{mg} / \mathrm{ml}$; Roche, Mannheim, Germany), Collagenase/Dispase (1 mg/ml; Roche), and Papain (25 U; Worthington Biochemical) for $1 \mathrm{~h}$ at $37^{\circ} \mathrm{C}$ in a shaking $\mathrm{CO}_{2}$ incubator $(200 \mathrm{rpm})$. Tissue homogenates were centrifuged at 1500 rpm for $5 \mathrm{~min}$ at $4^{\circ} \mathrm{C}$. The supernatant was discarded and the cells were resuspended in 70\% Percoll (GE Healthcare) and underlaid in 30\% Percoll. This gradient was centrifuged at $500 \mathrm{~g}$ for $20 \mathrm{~min}$ at $21^{\circ} \mathrm{C}$. Myelin was removed by suction and cells at the interface were collected. Cells were washed and blocked with mouse Fc Block (BioLegend) before staining with primary antibody-conjugated fluorophores: CD45-eF450, CD11bAPCeF780, Ly6C-APC (all BioLegend). For live/dead discrimination, a fixable viability dye, Zombie Aqua (BioLegend), was diluted at 1:100 in Hank's balanced salt solution (HBSS; Invitrogen). Cells were briefly fixed in $2 \%$ paraformaldehyde (PFA). Data were acquired on a LSRII using FACSDiva 6.0 (BD Biosciences) and analyzed using FlowJo (Treestar). The entirety of each sample was acquired on medium flow rate until zero events were obtained, providing absolute cell counts for each hemisphere. Resident microglia were identified as the $\mathrm{CD}_{11 \mathrm{~b}}{ }^{+} \mathrm{Ly}_{6 \mathrm{C}}{ }^{-}$ CD $45{ }^{\text {int }}$ population (Ritzel et al., 2019), whereas peripherally derived immune cells were identified as $\mathrm{CD} 45^{\mathrm{hi}}$ as described previously (Ritzel et al., 2018). Tissue and cell type matched fluorescence minus one (FMO) controls were used to determine the gating for each antibody. Before assessment on the cytometer, isolated cells were briefly probed to determine caspase- 1 activity and IL- $1 \beta$ production. Immediately following Percoll separation, cells were washed with PBS, and incubated with $1 \times$ FLICA solution at $37^{\circ} \mathrm{C}$ for $30 \mathrm{~min}$ according to the manufacturer's instructions (ImmunoChemistry Technologies). Cells were washed in FACS buffer, blocked, stained, and fixed as above. This assay employs the fluorescent inhibitor probe FAM-YVAD-FMK to label active caspase-1 enzyme in living cells (Lage et al., 2019). For Intracellular cytokine production analysis, before staining $1 \mu \mathrm{l}$ of GolgiPlug containing brefeldin A (BD Biosciences) was added to $800 \mu \mathrm{l}$ of complete RPMI, and cells were incubated for $2 \mathrm{~h}$ at $37^{\circ} \mathrm{C}\left(5 \% \mathrm{CO}_{2}\right)$. Cells were resuspended in Fc Block, stained for surface antigens, and washed in HBSS. Cells were vortexed and resuspended in $100 \mu \mathrm{l}$ of fixation/permeabilization solution (BD Biosciences) for $20 \mathrm{~min}$. Cells were then washed twice in $300 \mu \mathrm{l}$ of permeabilization/wash buffer (BD Biosciences), resuspended in IL- $1 \beta$ PerCPeF710 (BioLegend) for $30 \mathrm{~min}$, washed, and subsequently fixed, as described previously (Ritzel et al., 2018).

\section{Statistical analysis}

Blinding was performed as follows: the individual who administered drugs was blinded to treatment group and behavioral and stereological analyses were performed by individuals blinded to injury or treatment groups. Quantitative data were expressed as mean \pm SEM. Normality testing was performed and all data passed normality (D'Agostino and Pearson omnibus normality test), and therefore parametric statistical analysis was performed. BW and acquisition days of the MWM was analyzed by one-way repeated-measures ANOVA to determine the interactions of time and groups, followed by post hoc adjustments using a Bonferroni's multiple-comparisons test. The MWM search strategy was analyzed using a $\chi^{2}$ analysis. Y maze, NOR, Rotorod, the MWM Probe trial, qRT-PCR, flow cytometry, microglia morphology and neuronal cell loss was analyzed by one-way ANOVA, followed by post hoc adjustments using a Bonferroni's multiple-comparisons test. Stereological data for lesion volume and immunofluorescence staining was analyzed using a Student's $t$ test. Statistical analyses were performed using GraphPad
Table 1. Microglial depletion and repopulation do not negatively affect animal behavior in noninjured mice, including tests of anxiety, motor, and cognitive function

\begin{tabular}{|c|c|c|c|}
\hline & $\begin{array}{l}\text { Sham + } \\
\text { vehicle } \\
(n=10)\end{array}$ & $\begin{array}{l}\text { Sham + } \\
\text { PLX5622 } \\
(n=10)\end{array}$ & $p$-value \\
\hline BW WPI 0 (ff) & $3.4 \pm 1.5$ & $1.7 \pm 0.9$ & $>0.999^{a}$ \\
\hline BW WPI 1 (ff) & $2.8 \pm 0.9$ & $5.7 \pm 2.9$ & $0.9937^{a}$ \\
\hline BW WPI 2 (ff) & $5.2 \pm 2.1$ & $8.7 \pm 2.4$ & $0.9677^{a}$ \\
\hline BW WPI 3 (ff) & $2.0 \pm 1.2$ & $5.2 \pm 2.3$ & $0.9822^{a}$ \\
\hline BW WPI 4 (ff) & $5.6 \pm 1.5$ & $8.7 \pm 2.4$ & $0.9884^{a}$ \\
\hline BW WPI 5 (ff) & $4.3 \pm 1.5$ & $10.8 \pm 1.4$ & $0.2911^{a}$ \\
\hline BW WPI 6 (ff) & $5.9 \pm 2.7$ & $6.6 \pm 1.9$ & $>0.999^{a}$ \\
\hline BW WPI 7 (ff) & $5.6 \pm 2.1$ & $9.6 \pm 3.1$ & $0.9151^{a}$ \\
\hline BW WPI 8 (ff) & $4.4 \pm 2.3$ & $11.6 \pm 2.3$ & $0.1688^{a}$ \\
\hline BW WPI 9 (ff) & $8.9 \pm 1.9$ & $12.2 \pm 1.8$ & $0.9770^{a}$ \\
\hline BW WPI 10 (ff) & $8.8 \pm 1.2$ & $11.2 \pm 1.8$ & $0.9988^{a}$ \\
\hline BW WPI 11 (ff) & $10.4 \pm 2.8$ & $10.9 \pm 2.0$ & $>0.999^{a}$ \\
\hline BW WPI 12 (ff) & $10.2 \pm 2.3$ & $75.1 \pm 4.4$ & $>0.999^{a}$ \\
\hline Y maze (\% spontaneous alternation) & $71.1 \pm 3.7$ & $71.4 \pm 3.0$ & $0.9507^{b}$ \\
\hline NOR (\% preference index) & $78.0 \pm 6.4$ & $68.3 \pm 3.6$ & $0.2532^{b}$ \\
\hline MWM acquisition day 1 (time to escape) & $73.9 \pm 4.1$ & $75.1 \pm 4.4$ & $>0.999^{a}$ \\
\hline MWM acquisition day 2 (time to escape) & $46.1 \pm 7.7$ & $56.0 \pm 6.9$ & $0.996^{a}$ \\
\hline MWM acquisition day 3 (time to escape) & $56.4 \pm 6.9$ & $58.1 \pm 4.9$ & $>0.999^{a}$ \\
\hline MWM acquisition day 4 (time to escape) & $48.6 \pm 6.2$ & $40.1 \pm 5.6$ & $>0.999^{a}$ \\
\hline MWM probe test (time in escape quadrant) & $26.7 \pm 4.1$ & $23.2 \pm 2.9$ & $0.3218^{c}$ \\
\hline
\end{tabular}

Neurobehavioral testing confirmed that sham + vehicle and sham + PLX6522-treated C57BL/6J mice had equal performance in BW, Y-maze, NOR, and MWM tests. Summary statistical data and $p$-values are shown for each neurobehavioral test ( $n=10 /$ group).

WPI, weeks postinjury; ff, foot faults.

${ }^{a}$ Sidak's multiple-comparisons test.

${ }^{b}$ Mann-Whitney test.

Unpaired $t$ test.

Prism Program, Version 8 for Windows. $p<0.05$ was considered statistically significant.

\section{Results}

\section{Delayed depletion of microglia with CSF1R inhibitor after TBI improves long-term motor and cognitive function recovery}

Microglia are critically dependent on CSF1R signaling for their survival (Elmore et al., 2014). PLX5622 is an orally bioavailable, brain-penetrant CSF1R inhibitor, that is able to achieve robust brain-wide microglia elimination (Spangenberg et al., 2019). To demonstrate its utility in our preclinical study, PLX5622 (1200 $\mathrm{ppm}$ ) was formulated in rodent chow and administered to adult male C57BL/6J mice for $7 \mathrm{~d}$. When compared with control levels in Veh-treated mice, oral PLX5622 treatment led to almost complete microglial elimination (95\% reduction) within $7 \mathrm{~d}$, as demonstrated by loss of microglial staining by flow cytometry $\left(\mathrm{CD} 11 \mathrm{~b}^{+} / \mathrm{CD} 45^{\mathrm{int}}\right)$ and immunofluorescence $\left(\mathrm{P} 2 \mathrm{Y} 12^{+}\right)$analyses (Fig. $1 B, C$ ). Returning PLX5622-treated mice to normal chow resulted in microglial repopulation in the brain within $7 \mathrm{~d}$, such that numbers of repopulated microglia were not different to levels in Veh-treated control brain (Fig. 1C).

To investigate the effect of temporary depletion of microglia during the chronic stages of TBI on long-term neurological recovery, Sham and moderate-level CCI mice were orally administered PLX5622 (1200 ppm) or Veh in chow for 1-week starting at 4 weeks postinjury (WPI). All mice were returned to normal chow at 5 WPI to allow for microglial repopulation, and comprehensive motor and cognitive functional testing was performed from 8 to 12 WPI (Fig. 1A). Microglial depletion does not negatively affect animal behavior in noninjured mice, including tests 

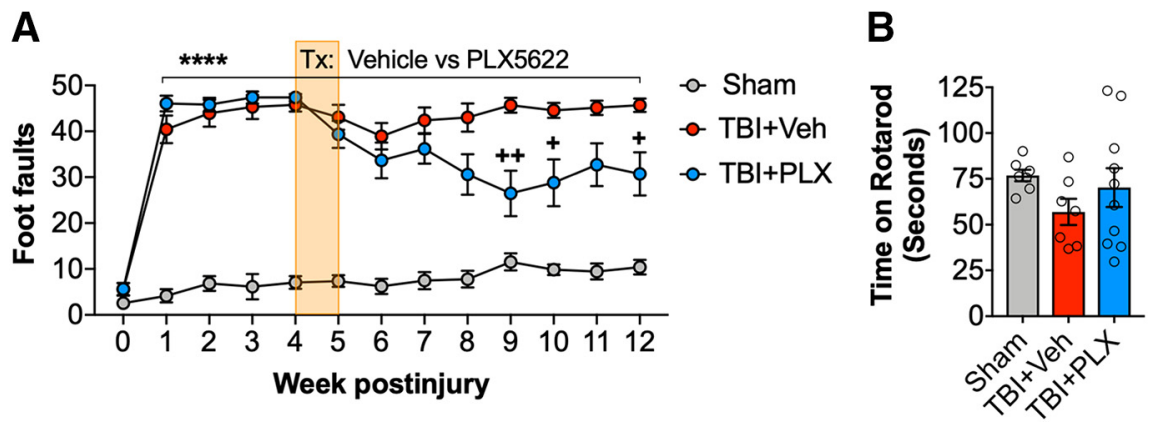

Figure 2. Delayed depletion of microglia starting at 4 weeks postinjury improves long-term motor function recovery. A, Delayed depletion of microglia using the CSF1R inhibitor, PLX5622, significantly reduced long-term TBI-induced fine-motor impairments (number of foot faults in a BW task) at 9, 10, and 12 WPI. $B, A t 12$ WPI, TBI + Veh-treated animals spent less time on the accelerating rod when compared with Sham counterparts. ${ }^{* * * *} p<0.0001$ vs Sham; $+p<0.05,++p<0.01$ vs TBI + Veh. Data expressed as mean \pm SEM ( $n=7-20 /$ group).

of anxiety, motor-function, and cognition (Rice et al., 2015; Spangenberg et al., 2019). Our neurobehavioral testing confirmed that Sham + Veh and Sham + PLX6522-treated C57BL/6J mice had equal performance in BW, Y-maze, NOR and MWM tests (Table 1). Therefore, Veh- and PLX5622-treated Sham mice were combined (denoted Sham) for all behavioral analyses.

TBI induces long-term deficits in fine motor coordination in a BW task. Before microglia depletion at 4 WPI, TBI mice had $42.8 \pm 2.0 \mathrm{ff}$ (mean $\pm \mathrm{SEM}$ ) when compared with $7.1 \pm 1.4 \mathrm{ff}$ in Sham mice ( $p<0.001$ vs Sham; Fig. $2 A)$. Sham and TBI mice were then randomized into cohorts that received PLX5622 or Veh for 1-week to deplete and subsequently repopulate microglia, and mice were tested weekly through the end of study. TBIinduced deficits in motor coordination persisted in Veh-treated TBI mice at $12 \mathrm{WPI}(\mathrm{TBI}+\mathrm{Veh}=45.7 \pm 1.4 \mathrm{ff}$ vs Sham $=10.6 \pm$ $1.5 \mathrm{ff} ; \mathrm{TBI}$ effect, $F_{(2,611)}=633.4, p<0.001 ; p<0.001$ vs Sham; Fig. 2A). PLX5622 treatment significantly improved postinjury BW performance $\left(F_{(24,611)}=6.93, p<0.001\right)$. When compared with deficits in Veh-treated TBI mice, TBI+PLX5562-treated mice had reduced ff at $8(\mathrm{TBI}+\mathrm{Veh}=43.0 \pm 3.1 \mathrm{ff}$ vs $\mathrm{TBI}+\mathrm{PLX}=30.6 \pm 4.4 \mathrm{ff} ; p=0.09), 10(\mathrm{TBI}+\mathrm{Veh}=44.6 \pm 1.6$ ff vs TBI+PLX $=28.8 \pm 5.1 \mathrm{ff} ; p=0.04)$, and $12(\mathrm{TBI}+\mathrm{Veh}=$ $45.7 \pm 1.4 \mathrm{ff}$ vs TBI + PLX $=30.7 \pm 4.7 ; p=0.04$ ) WPI. Motor balance was also evaluated using an accelerating rotarod task at 12 WPI. Veh-treated TBI mice spent less time on the accelerating rotorod than Sham mice $(\mathrm{TBI}+\mathrm{Veh}=57.0 \pm 7.2 \mathrm{~s}$ vs Sham $=$ $76.9 \pm 3.2$ s; Fig. 2B). In contrast, PLX5622-treated TBI mice spent more time on the rotarod (TBI+PLX $=70.3 \pm 10.6 \mathrm{~s})$ than Veh-treated TBI mice, but differences did not reach statistical significance.

We next investigated cognitive function using a Y-maze task that assesses hippocampal-dependent working memory. When compared with the percentage spontaneous alternations in Sham mice $(71.3 \pm 2.3 \%)$, Veh-treated TBI mice had decreased spontaneous alternations in the task, indicative of working memory deficits $\left(60.4 \pm 3.6 \%\right.$; TBI effect, $F_{(2,48)}=3.926 ; p=0.02$ vs Sham; Fig. $3 A$ ). In contrast, PLX5562-treated TBI mice (68.5 \pm 3.4) had similar performance as Sham mice, indicating improved spatial working memory following PLX5622 treatment. Nonspatial hippocampal-mediated memory was also tested using a NOR task at 11 WPI. During the familiar phase of the test, there was no difference between Sham, Veh-treated or PLX5622-treated TBI mice with regards to time spent with either the right or left objects, indicating no side preference (Fig. 3B). Twenty-four hours later, mice were retested with a novel object. When compared with Sham mice (78.0 \pm $5.3 \%)$, Veh-treated TBI mice spent significantly less time with the novel object $\left(46.0 \pm 6.0 \%\right.$; TBI effect, $F_{(2,28)}=5.914$; $p=0.007$ vs Sham; Fig. $3 B)$. PLX5622treated TBI spent an increased amount of time with the novel object (59.0 \pm 7.6) when compared with the Vehtreated TBI group; however, differences did not reach statistical significance.

We performed the MWM at 12 WPI to assess spatial learning and memory. Oneway repeated-measures ANOVA revealed an effect of time (acquisition day, AD; $\left.F_{(3,208)}=7.868, p<0.001\right)$, and PLX5622 treatment $\left(F_{(2,208)}=41.63, p<0.001\right)$, and an interaction between both factors on latency to the escape platform during the test $\left(F_{(6,208)}=2.756, p=0.04\right)$. When compared with Sham mice, both Veh-treated and PLX5622-treated TBI mice had increased latency times during $\operatorname{AD} 2(p=0.01$ vs Sham), $\operatorname{AD} 3(p=$ 0.04 vs Sham), and AD4 ( $p<0.001$ vs Sham) (Fig. $3 C$ ). There were no differences in escape latencies between Veh-treated and PLX5622-treated TBI mice at any time point. Twenty-four hours later, retention memory was assessed using a probe trial. There was a significant effect of PLX5622 treatment on percentage time spent in the escape quadrant during the probe trial $\left(F_{(2,47)}=\right.$ $11.14, p<0.001$; Fig. $3 D$ ). Veh-treated TBI mice spent less time in the escape quadrant than Sham mice ( $p<0.001$ vs Sham). In contrast, PLX5622 treatment reversed TBI-induced retention memory deficits, and PLX5622-treated TBI mice spent more time in the escape quadrant than Veh-treated TBI mice $(p=0.04$ vs TBI+Veh; Fig. $3 D$ ). There were no differences between groups for swim speeds (Fig. $3 E$ ), indicating that cognitive improvements were independent of deficits in gross locomotor activity. Finally, to investigate search strategies used by mice to find the hidden platform, the swim pattern for each mouse was analyzed and assigned a search strategy (i.e., spatial, nonspatial systematic, or repetitive looping) as described previously (Brody and Holtzman, 2006; Byrnes et al., 2012; Zhao et al., 2012). Veh-treated TBI mice primarily used repetitive looping search strategies (70\%) to find the submerged platform when compared with Sham mice (25\% looping strategy; Fig. $3 F$ ). In contrast, Sham and PLX5622treated TBI mice predominantly used a systematic search strategy $($ Sham $=65 \%$; TBI + PLX $=47 \%)$ when compared with Vehtreated TBI mice ( $30 \%$ systematic search strategy; $p<0.001, x^{2}=$ 38.43).

\section{Delayed depletion of microglia with CSF1R inhibitor after TBI reduces histological markers of neurodegeneration and chronic microglial activation}

To investigate the effect of temporary depletion of microglia during the chronic stages of TBI on neuropathology, lesion volume and neuronal loss was quantified at 3 months postinjury. As expected, TBI produced a large cortical lesion in the Veh-treated group $\left(7.26 \pm 0.76 \mathrm{~mm}^{3}\right.$; Fig. $\left.4 A, B\right)$. In contrast, PLX5622 treatment reduced the cortical lesion $\left(5.55 \pm 0.52 \mathrm{~mm}^{3} ; t_{(19)}=1.744\right.$, $p=0.04$ vs TBI + Veh $). T B I$ also induced significant neuronal loss in the ipsilateral cortex $\left(F_{(2,21)}=9.560, p<0.001\right.$; Fig. $\left.4 C\right)$ and the dentate gyrus (DG) of the hippocampus $\left(F_{(2,19)}=6.417, p=\right.$ 0.007; Fig. 4D). Post hoc analysis revealed that TBI resulted in neuronal loss in cortex ( $p=0.003$ vs Sham) and DG ( $p=0.02$ vs Sham), and that PLX5622 treatment reduced neuronal loss in 
both the cortex $(p=0.05$ vs TBI + Veh; Fig. $4 C)$ and DG ( $p=0.03$ vs TBI+Veh; Fig. $4 D$ ) at 3 months postinjury.

We next performed stereological assessment of Iba1 ${ }^{+}$cells to quantify microglial cell number and activation state in the perilesional cortex at 3 months postinjury, as described previously (Byrnes et al., 2012; Loane et al., 2014). Despite having an equal number of total Iba ${ }^{+}$cells in each group (data not shown), there was an effect of TBI on the number of ramified $\left(F_{(2,30)}=4.718, p=0.01\right.$; Fig. $\left.4 E\right)$ and hypertrophic/bushy microglia $\left(F_{(2,19)}=\right.$ $6.884, p=0.005$; Fig. $4 E)$. There were reduced numbers of Iba ${ }^{+}$ramified microglia in the ipsilateral cortex of Veh-treated TBI mice compared with Sham mice $(p<$ 0.05; Fig. 4E). By contrast, PLX5622 treatment increased numbers of ramified microglia in the injured cortex $(p=0.04$, TBI+PLX vs TBI+Veh; Fig. $4 E$ ). TBI also increased the numbers of Ibal ${ }^{+}$hypertrophic/bushy microglia in the ipsilateral cortex of Veh-treated TBI mice compared with Sham mice ( $p=0.03$ vs Sham; Fig. $4 E)$. Notably, PLX5622 treatment reduced the numbers of hypertrophic/bushy $\mathrm{Ibal}^{+}$microglia in the injured cortex when compared with Veh-treated TBI mice $(p=0.03$, TBI + PLX vs TBI+Veh; Fig. 4E).

\section{Delayed depletion of microglia with CSF1R inhibitor alters cortical transcription patterns related to neuroinflammation, oxidative stress, apoptosis, and neuroplasticity} We then sought to define network-level changes in key components of the cortical transcriptome after TBI by analyzing perilesional cortical tissue using a Nanostring Neuropathology panel that evaluates gene expression across fundamental pathways of neurodegeneration, including neurotransmission, neuron-glia interaction, neuroplasticity, cell structure integrity, neuroinflammation and metabolism. PLSDA and hierarchical clustering were used to identify differentially expressed genes between the four treatment groups. PLSDA allows for semisupervised classification of different treatment groups and determination of signature genes of each experimental variable. In our model, principal component (PC1) captured the variation caused by TBI, while PC2 captured variation caused by PLX5622 treatment (Fig. 5A). The PLSDA scores plot shows that gene expression signatures from PCs 1-2 clustered mice from each of the four treatment groups as expected: Group 1 (Sham+Veh), Group 2 (TBI+Veh), Group 3 (Sham+PLX5622), and Group 4 (TBI+PLX5622; Fig. 5A). The signature genes for each PC are shown in the extended data (Fig. 5-1A, available at https:// doi.org/10.1523/JNEUROSCI.2402-19.2020.f5-1), and the
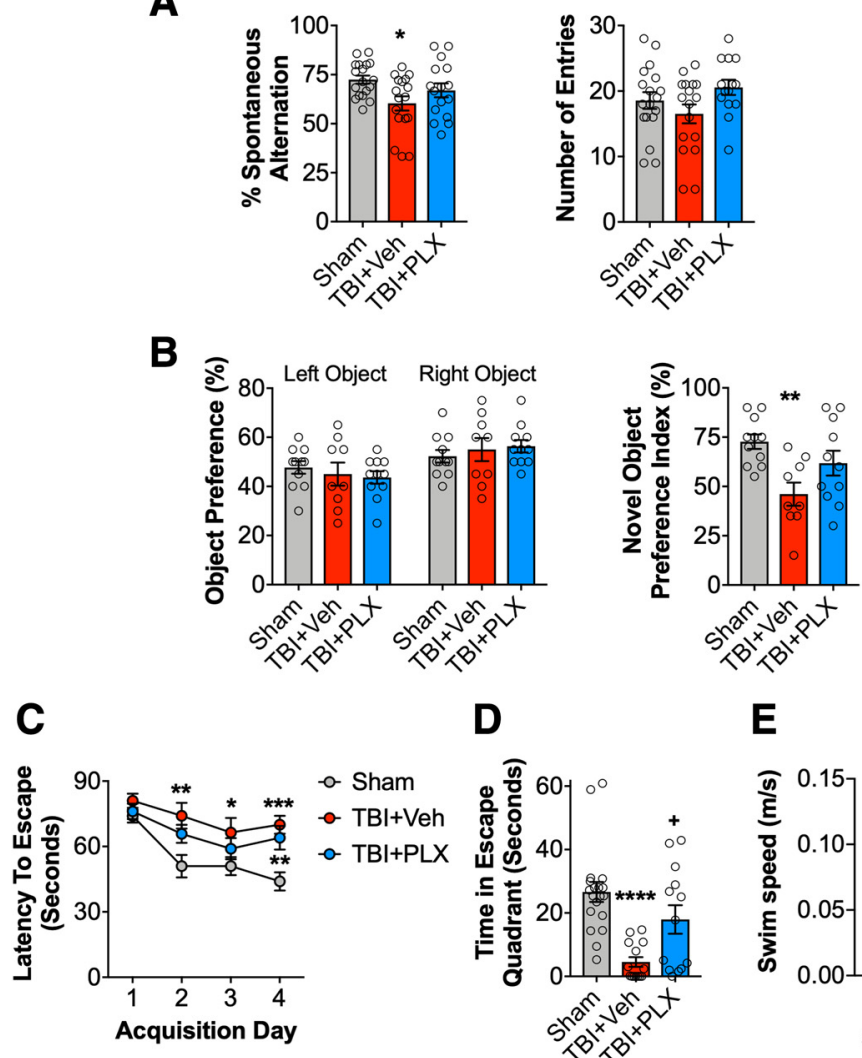

D
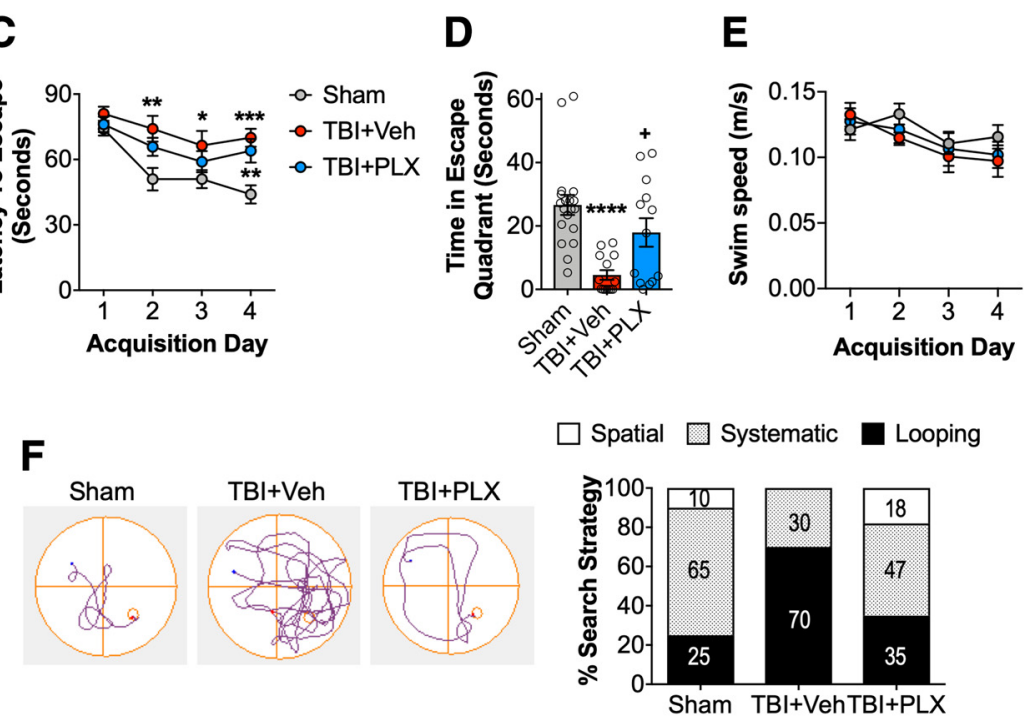

Figure 3. Delayed depletion of microglia starting at 4 weeks postinjury improves cognitive function recovery. $\boldsymbol{A}, \mathrm{At} 10 \mathrm{WPI}, \mathrm{TBI}$ significantly decreased percentage spontaneous alterations in the Y-maze task in TBI+Veh-treated animals, when compared with Sham. TBI+PLX5622-treated animals had increased percentage spontaneous alterations, equal to levels in Sham. B, At 11 WPI, TBI significantly decreased percentage time spent with the novel object in a novel object task in TBI+Veh-treated animals, when compared with Sham. TBI+PLX5622-treated animals spent increased time with the novel objects, similar to levels of Sham. $\boldsymbol{C}$, In the MWM task at 12 WPI, TBI+Veh- and TBI + PLX5622-treated animals required significantly increased time to locate the hidden submerged escape platform on acquisition days 2-4, when compared with Sham. There was no significant difference in escape latencies between $\mathrm{TBI}+$ Veh- and TBI + PLX5622-treated animals. $\boldsymbol{D}$, In the probe trial, TBI + Veh-treated animals spent significantly less time in the escape quadrant, when compared with Sham. TBI+PLX5622-treated animals spent significantly more time in the escape quadrant, when compared with TBI + Veh-treated animals. $E, T B I+$ Veh-treated and TBI + PLX5622-treated animals had similar swim speeds to that of Sham animals during the acquisition days of the MWM. $\boldsymbol{F}$, Escape strategy used during the probe trial was assessed, and the percentage composition for each strategy (spatial, systematic, and looping) demonstrated that $\mathrm{TBI}+$ Veh-treated animals used increased looping strategies, and decreased systematic and spatial search strategies when compared with Sham and TBI + PLX5622-treated animals. ${ }^{*} p<0.05,{ }^{* *} p<0.01,{ }^{* * *} p<0.001,{ }^{* * *} p<0.0001$ vs Sham; $+p<$ 0.05 vs TBI + Veh. Data expressed as mean \pm SEM ( $n=7-20 /$ group $)$.

loadings indicated the influence of each gene on signatures of TBI (PC1) or PLX5622 treatment (PC2). Genes with more positive loadings on PC1 were upregulated with TBI while negative loadings on PC1 were downregulated by TBI. Overall model performance was very good for all treatment groups (Fig. 5-1B, available at https://doi.org/10.1523/JNEUROSCI.2402-19.2020. f5-1) with classification errors ranging from $84.56 \%$ (Group 1) to $98.58 \%$ (Group 4). Group 4 was the least effectively classified due to the large intragroup variance in gene expression, suggesting that TBI+PLX5622 outcomes were more heteroge- 
A
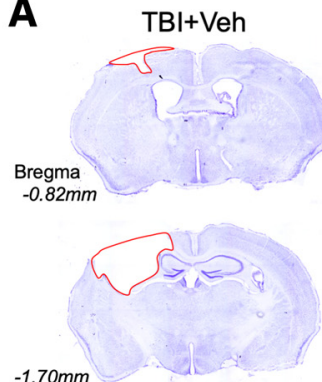

$-1.70 \mathrm{~mm}$

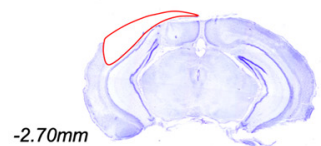

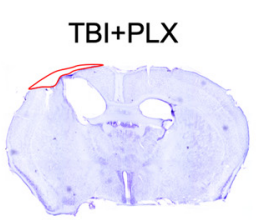
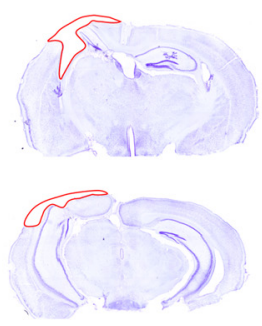

B
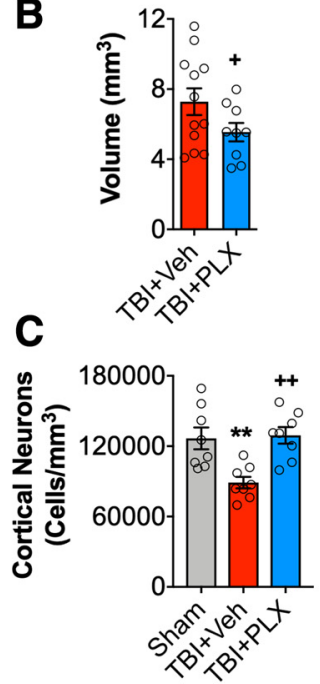

$\mathbf{E}$

D
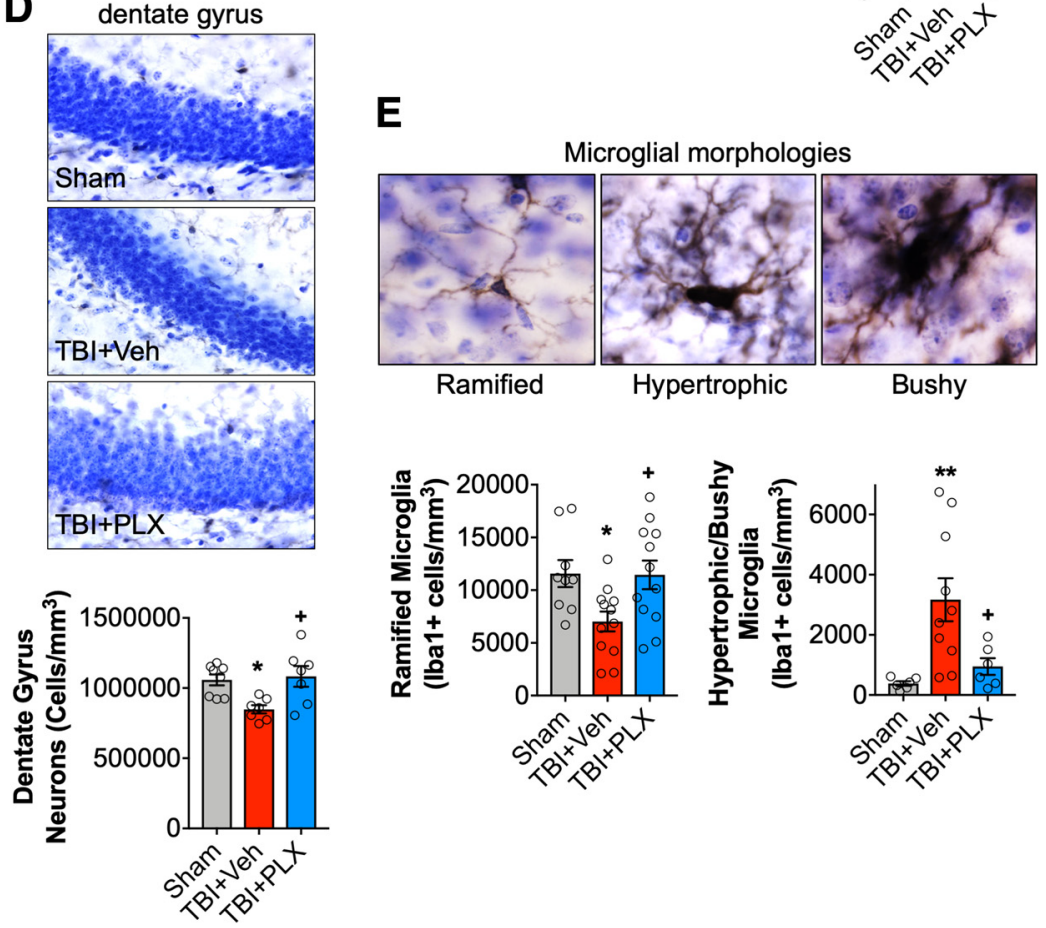

Ramified

Hypertrophic

Bushy
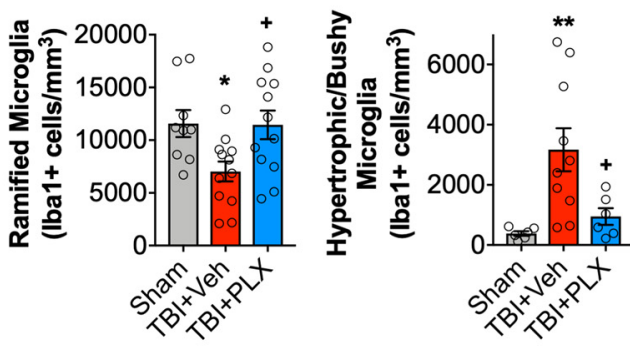

Figure 4. Delayed depletion of microglia starting at 4 weeks postinjury reduces chronic neurodegeneration and alters microglial morphology. $\boldsymbol{A}$, Representative images of the cortical lesion in TBI+Veh- and TBI+PLX5622-treated animals at 3 months postinjury. $\boldsymbol{B}$, Stereological analysis demonstrated that TBI+PLX5622-treated animals had a decreased lesion volume, when compared with $\mathrm{TBI}+$ Veh-treated animals. C, Stereological analysis demonstrated that TBI significantly increased neuron loss in ipsilateral cortex, when compared with Sham. PLX5622 treatment attenuated cortical neuron loss such that TBI+PLX5622treated animals had increased numbers of surviving neurons in cortex compared with TBI+Veh-treated animals. $\boldsymbol{D}$, Representative images of cresyl violet stained neurons in the dentate gyrus (DG) of hippocampus. Stereological analysis demonstrated that TBI increased DG neuron loss, when compared with Sham. In contrast, TBI+PLX5622-treated animals had increased numbers of surviving neurons in DG compared with $\mathrm{TBI}+$ Veh-treated animals. $\boldsymbol{E}$, Representative images of Iba1+ microglia displaying ramified, hypertrophic, and bushy cellular morphologies. Stereological analysis demonstrated that TBI + Veh-treated animals had decreased numbers of ramified and increased hypertrophic/bushy lba ${ }^{+}$microglia in injured cortex, when compared with Sham. In contrast, $\mathrm{TBI}+\mathrm{PLX} 5622$-treated animals had increased ramified and decreased hypertrophic/bushy lba1 ${ }^{+}$microglia when compared with TBI+Veh-treated animals. ${ }^{*} p<0.05,{ }^{* *} p<0.01$ vs Sham; $+p<0.05,++p<0.01$ vs TBI +Veh. Data expressed as mean \pm SEM ( $n=8-12 /$ group).

neous at the level of gene expression. The high classification accuracies for all 4 experimental groups indicated that the first 2 PCs captured signature genes changed by TBI and PLX5622 treatment.

Differential expression analyses were performed using NanoStringDiff to identify statistically significant differences in gene expression between groups. We focused on differences in Sham +Veh vs Sham + PLX5622 groups, and TBI+Veh vs TBI+
PLX5622 groups. Analysis of inflammation and immune related genes identified a cluster of microglial-related genes $(\mathrm{MgCl})$ that were elevated in TBI+Veh compared with Sham+Veh and Sham+PLX5622 groups (Fig. 5B). Critically, the TBI+ PLX5622 group had reduced expression of $\mathrm{MgCl}$ genes compared with TBI+Veh. $\mathrm{MgCl}$ upregulated genes included inflammatory receptors (e.g., $C d 33, C \times 3 c r 1, C d 44$ and $\mathrm{Cxcr} 4$ ), the IFN $\gamma$-induced immunoproteasome gene Psmb9, and the TGF $\beta$ dependent microglia-specific gene Fcrls. The NADPH oxidase-related genes, $C y b b$ and $N c f 1$, were also upregulated in $\mathrm{TBI}+\mathrm{Veh}$ group, and attenuated in the TBI+PLX5622 group. We next examined 2 clusters of genes involved in oxidative stress responses that were changed by PLX5622 treatment (Fig. 5C). The first cluster $(O x C 1)$ included genes upregulated by TBI, with highest expression in TBI+Veh group that was decreased by PLX5622 treatment (e.g., Nme5, Gsr, Hgf, $\mathrm{Hfe} 2 \mathrm{l2})$. Another cluster $(\mathrm{O} x \mathrm{C} 2)$ of genes (Ppargcla, Nos3, Gucy1b3, Atp7a) demonstrated an inverse trend: they had increased expression in the TBI+PLX5622 group when compared with the TBI+Veh group. Apoptosis-related genes were also evaluated (Fig. 5D). Broadly, each experimental group had a distinct pattern of apoptotic gene expression. PLX5622 acted partially by reducing expression of key caspases (Casp6, Casp7) in injured cortex. Treatment with PLX5622 also decreased the upstream proapoptotic genes $B a x$, a mitochondrial membrane permeator. Neuroplasticity genes were also assessed (Fig. 5E). The most highly expressed genes in Sham +Veh were most downregulated in the TBI+PLX5622 group, indicating that PLX5622 treatment does not return neuroplasticity gene expression to a preinjured state.

Finally, focused analysis of transcript counts for selected genes related to NLRP3 inflammasome (Illr1, Casp1, $P 2 r x 7, N l r p 3)$ and NADPH oxidase (Cybb, Ncf1) pathways demonstrated that key genes in both inflammatory pathways are reduced by PLX5622 treatment during chronic TBI (Fig. 5F). We confirmed these microglial-related inflammatory pathways in injured cortex using real-time PCR and immunofluorescence imaging. Short-term depletion and subsequent repopulation of microglia during the chronic stages of TBI by PLX5622 treatment decreased TBI-induced upregulation of NLRP3 inflammasome related genes (Nlrp3, Casp1, and Illb; Fig. $6 A$ ), and NADPH oxidase genes (Cybb, Cyba, Ncf1, Ncf4; Fig. $6 B$ ) in RNA isolated from the perilesional cortex. Immunofluorescence imaging confirmed $\mathrm{NADPH}$ oxidase $\left(\mathrm{NOX}_{2}{ }^{+}\right.$) expression in reactive microglia $\left(\mathrm{Ibal}^{+} / \mathrm{CD} 8^{+}\right)$in the injured cortex at 3 months postin- 


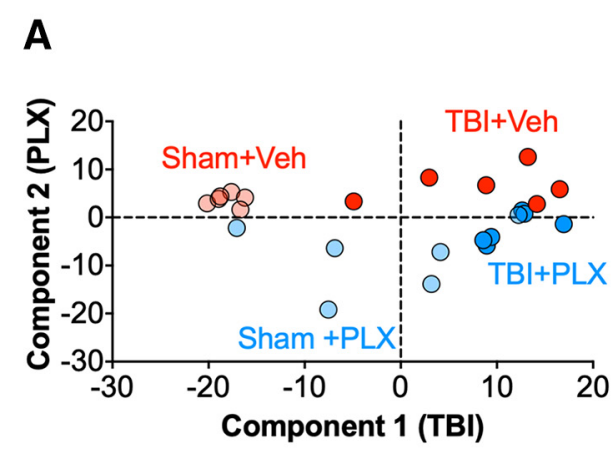

B Inflammation \& Immune related
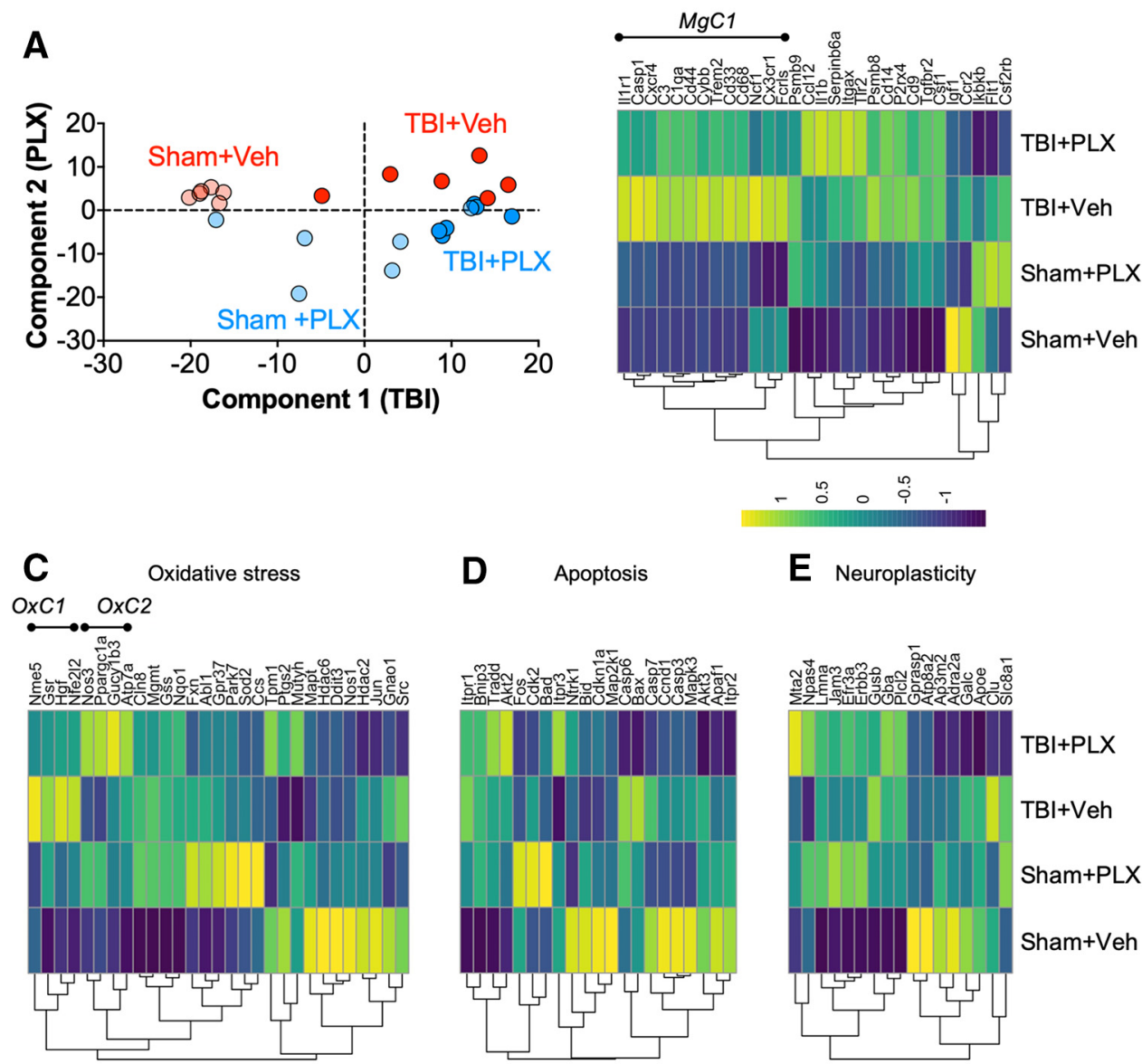

D Apoptosis

E Neuroplasticity
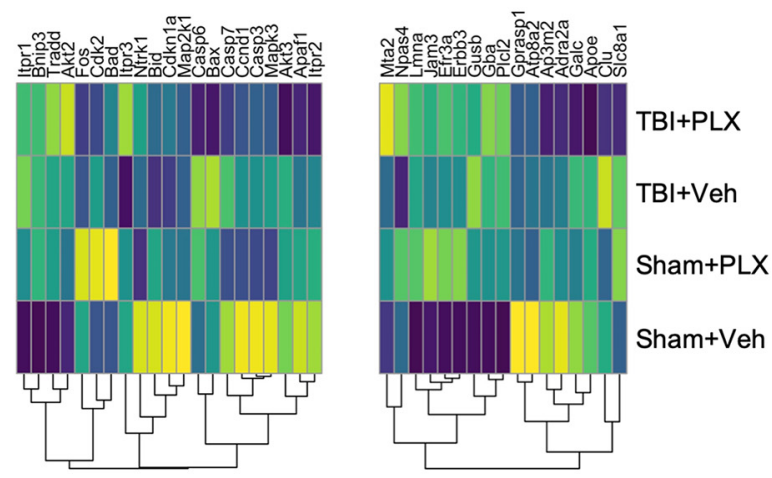

$\mathbf{F}$

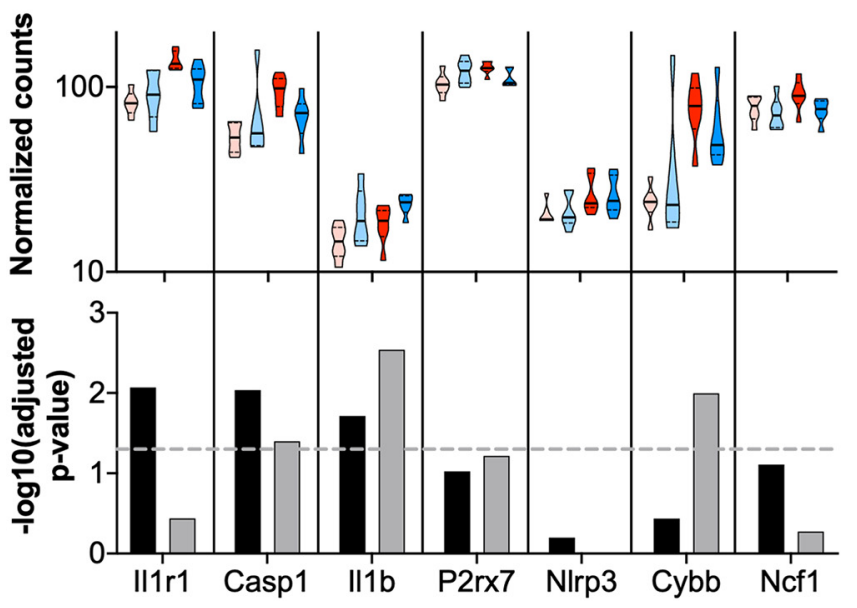

$\mathrm{TB} \mid+$ Veh vs TBI+PLX

$\square$ Sham+Veh vs

Sham+PLX

Figure 5. Delayed depletion of microglia during chronic TBI alters neuroinflammation, oxidative stress, apoptosis, and neuroplasticity pathways in the cortex. A Nanostring Neuropathology Panel was used to assess cortical transcriptional patterns at 2 months postinjury. A, A PLSDA model was generated to classify each of the 4 treatment groups: Group 1 (Sham + Veh), Group 2 (TBI + Veh), Group 3 (Sham + PLX5622), and Group 4 (TBI+PLX5622). Principal components (PC) 1 and 2 identified key genes changed by TBI and PLX5622 treatment, respectively. $\boldsymbol{B}$, Hierarchical clustering of differentially expressed genes was performed on different functional annotations. TBl induced a large microglial-related gene cluster $(\mathrm{Mg}(1)$ associated with inflammation and immune function, representing the inflammatory state induced by TBI. C, Analysis of oxidative stress genes identified 2 clusters of genes altered by PLX5622 treatment. The first cluster (OXC1) included genes that were increased after TBI, and were significantly reduced by TBI+PLX5622 treatment. The other cluster (0xC2) was significantly increased in TBI+PLX5622 animals when compared with the TBI + Veh animals. D, Clustering analysis of apoptosis-related genes showed that TBI increased expression of the mitochondrial membrane permeator, Bax, which was decreased by PLX5622 treatment. $\boldsymbol{E}$, Clustering analysis of neuroplasticity genes showed that the most highly expressed neuroplasticity genes in Sham + Veh were downregulated by TBI, including in TBI + PLX5622-treated animals. This indicated that PLX5622 treatment did not return neuroplasticity gene expression to a preinjured state. $\boldsymbol{F}$, Analysis of transcript counts (Violin plots and -log10 adjusted P value assessment) for selected genes related to NLRP3 inflammasome (II1r1, Casp1, $1 / 16$ P2rx7, NIrp3) and NADPH oxidase (Cybb, Ncf1) pathways demonstrated that key genes in both inflammatory pathways were upregulated by TBI, and subsequently reduced by PLX5622 treatment. ( $n=8$-9/group). (Fig. 5-1, available at https://doi.org/10.1523/JNEUROSCI.2402-19.2020.f5-1). 

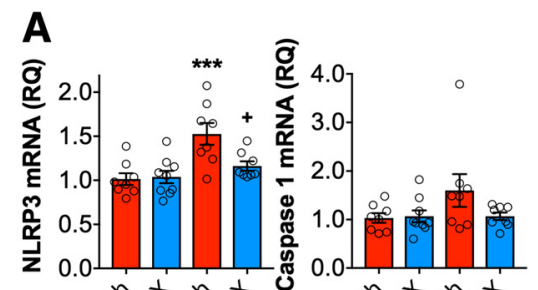

B
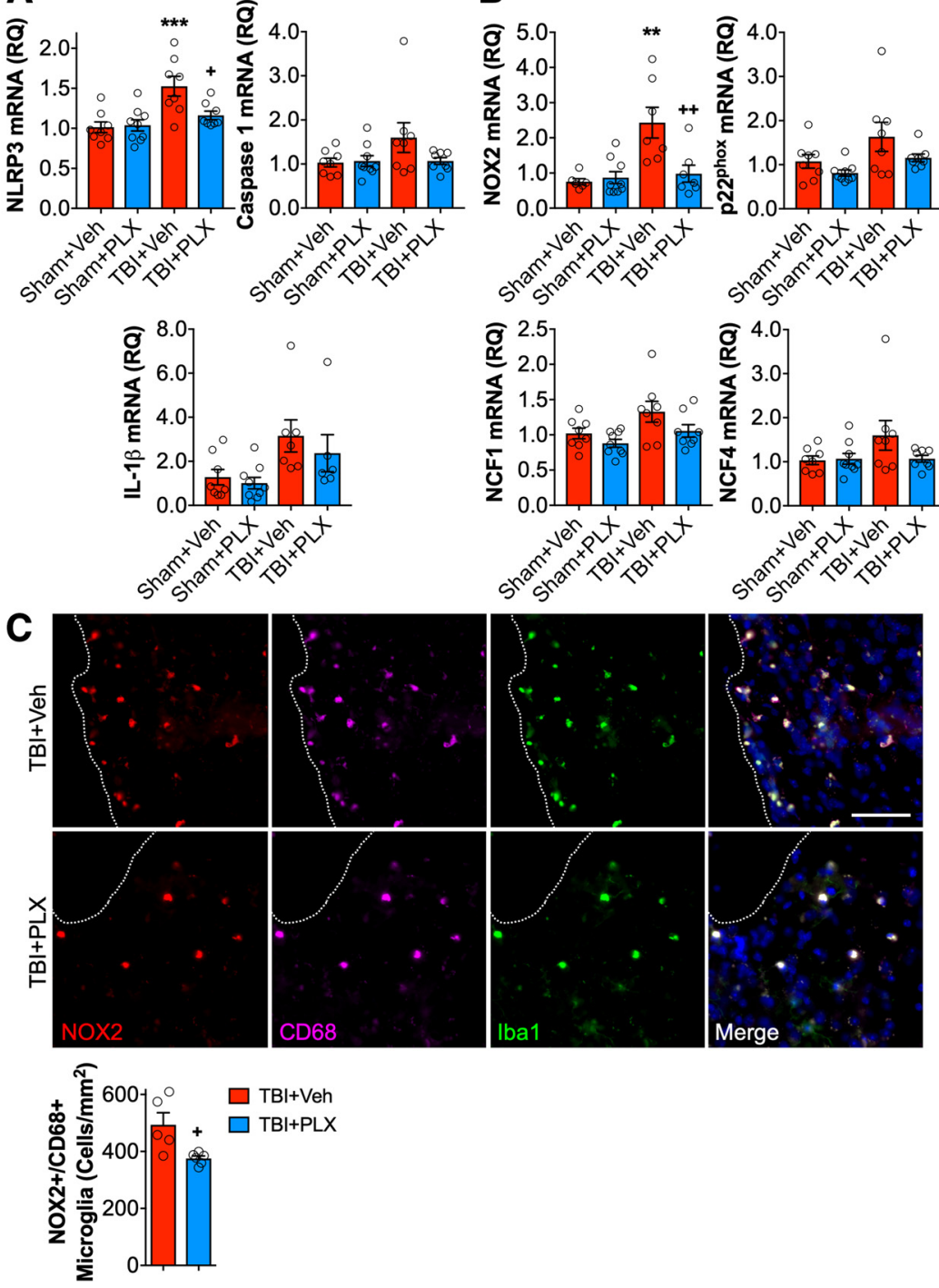

Figure 6. Delayed depletion of microglia during chronic TBI decreases NLRP3 inflammasome and NOX2 expression in the injured cortex. A, Analysis of ipsilateral cortical tissue at 2 months postinjury demonstrated that TBI increased N/rp3, Casp 1, and I/16 mRNA. The TBI-induced increase in NIrp3 mRNA was significantly reduced by PLX5622 treatment. $\boldsymbol{B}$, TBI increased NADPH oxidase subunits, $\mathrm{Cy} b b, \mathrm{Cy} b a, \mathrm{Ncf1}$, and Ncf4 mRNA in injured cortex. The TBI-induced increase in Cybb mRNA was significantly reduced by PLX5622 treatment. $\boldsymbol{C}$, Immunofluorescence analysis of reactive microglia (NOX2 ${ }^{+}$, red; $\mathrm{CD} 68^{+}{ }^{+}$, cyan; $\mathrm{Iba} 1^{+}$, green) in the injured cortex at 3 months postinjury demonstrated that delayed depletion of microglia decreased the number of N0X2 ${ }^{+}$reactive microglia when compared with levels in TBI+Veh-treated animals. The dotted line represents the lesion boundary. Scale bar, 100 $\mu \mathrm{m} .{ }^{* *} p<0.01,{ }^{* * *} p<0.001$ vs Sham animals; $+p<0.05,++p<0.01$ vs TBI+Veh-treated animals. Data expressed as mean $\pm \operatorname{SEM}$ ( $n=6-9 /$ group).

jury (Fig. $6 C$ ). Notably, the number of $\mathrm{NOX}^{+} / \mathrm{CD} 68^{+}$reactive microglia was reduced in the injured cortex of PLX5622-treated TBI mice $\left(t_{(11)}=2.189 ; p=0.03\right.$ vs TBI+Veh; Fig. $\left.6 C\right)$.

Delayed depletion of microglia with CSF1R inhibitor reduced the inflammatory status of microglia at $\mathbf{2}$ months postinjury To better understand the cellular correlates related to chronic transcriptional changes in the injured cortex we evaluated inflammatory responses in microglia using flow cytometry. TBI increased absolute numbers of live $\mathrm{CD} 11 \mathrm{~b}^{+} / \mathrm{Ly}_{6 \mathrm{C}}-\mathrm{CD} 45^{\mathrm{int}} \mathrm{mi}-$ croglia at 2 months postinjury when compared with Sham levels (Fig. 7A); PLX6522 treatment had no effect on microglial cell numbers at this time point. The total number of infiltrating $\mathrm{CD} 45^{\text {hi }}$ leukocytes were also increased by TBI when compared
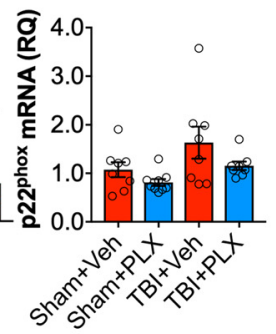

with Sham levels (Fig. 7A), but relative numbers were low $\left(291 \pm 44 \mathrm{CD} 45^{\text {hi }}\right.$ cells) compared with numbers of resident microglia (48474 $\pm 3108 \mathrm{CD} 45^{\text {int }}$ cells). Again, PLX5622 treatment had no effect on infiltrating $\mathrm{CD} 45^{\text {hi }}$ cell numbers. Microglial morphological features were examined including analysis of microglial size by forward scatter (FSC) and microglial cellular granularity by side scatter (SSC). Consistent with histological findings for hypertrophic/bushy $\mathrm{Ibal}^{+} \mathrm{mi}^{-}$ croglia (Fig. 4E), TBI increased microglial cell size $\left(\mathrm{FSC}^{+} ; F_{(2,15)}=10.02, p=0.04 \mathrm{vs}\right.$ Sham $)$ and granularity $\left(\mathrm{SSC}^{+} ; F_{(2,19)}=\right.$ $21.34, p=0.01$ vs Sham) when compared with levels in Sham mice (Fig. $7 B$ ). PLX5622 treatment reduced both size and granularity parameters in microglia $\left(\mathrm{FSC}^{+}: p=0.005\right.$ vs TBI+Veh; $\mathrm{SSC}^{+}:$ $p<0.001$ vs TBI+Veh). Assembly of NLRP3 inflammasome leads to caspase-1dependent release of proinflammatory IL-1 $\beta$ (Heneka et al., 2018), so we assessed caspase- 1 activity and IL- $1 \beta$ expression in microglia. Consistent with mRNA analysis (Fig. 6A), TBI increased caspase- 1 activity $\left(F_{(2,10)}=5.608, p=0.02\right.$ vs Sham; Fig. $7 C)$ and IL- $1 \beta$ production $\left(F_{(2,11)}=\right.$ $14.01, p<0.001$ vs Sham; Fig. $7 D)$ in microglia when compared with levels in Sham mice. Notably, PLX5622 treatment reduced microglial caspase- 1 activity and IL- $1 \beta$ production $(p=0.007$ vs TBI + Veh). Therefore, delayed depletion of microglia using PLX5622 attenuates key markers of NLRP3 inflammasome activation in microglia during chronic stages of TBI.

\section{Delayed depletion of microglia with \\ CSF1R inhibitor does not affect \\ posttraumatic astrocytic responses}

Astrocytes tune their reactivity to varying degrees of axonal injury, vascular disruption, ischemia, and inflammation (Burda et al., 2016). Furthermore, astrocytes and microglia communicate via cytokines and cell-surface markers during aging, neurodegeneration and CNS injury (Villacampa et al., 2015; Liddelow et al., 2017). Therefore, to determine the effect of delayed PLX5622 treatment after chronic TBI on astrocytes in the cortex we evaluated GFAP expression, a canonical marker of astrocytes. TBI increased GFAP mRNA expression in the cortex $\left(F_{(2,19)}=4.644, p=0.02\right)$ when compared with levels in Sham mice ( $p=0.02$ vs Sham; Fig. $8 A$ ). GFAP mRNA expression was also increased in PLX5622-treated TBI mice. In situ analysis of $\mathrm{GFAP}^{+}$astrocytes in the perilesional cortex at 3 months postinjury confirmed that TBI increased astrocyte cellular area $\left(F_{(1,18)}=29.99, p=0.04\right.$; Fig. $\left.8 B\right)$ and GFAP expression $\left(F_{(1,18)}=21.36, p=0.003\right.$; Fig. $\left.8 B\right)$. However, PLX5622 treatment did not alter TBI-induced changes in GFAP cellular distribution or expression levels. 


\section{Discussion}

Recent clinical studies have revised the outdated concept that TBI is an acute neurological disorder (Wilson et al., 2017). Instead, persistent and diffuse posttraumatic neuroinflammation can continue for many years in TBI patients (Ramlackhansingh et al., 2011; Johnson et al., 2013; Coughlin et al., 2015, 2017; Cherry et al., 2016). Microglia undergo changes in their activation profile during chronic neuroinflammation that may contribute to neurological deficits in many neurodegenerative diseases (Perry et al., 2010; Heneka et al., 2015). Epidemiological studies show that a prior history of TBI is associated with an increased incidence of Alzheimer's disease (AD) and non-AD forms of dementia (Mortimer et al., 1985; Dams-O'Connor et al., 2013; Gardner et al., 2014; Nordström et al., 2014; Wilson et al., 2017). Experimental evidence suggests that such neurodegeneration is associated with maladaptive transformation of microglia from a neurorestorative or neuroprotective phenotype to a dysfunctional neurotoxic phenotype (Loane and Kumar, 2016). Thus, chronic release of neurotoxic molecules from microglia, including reactive oxygen species and proinflammatory cytokines, may cause chronic progressive neuronal loss and/or white matter degeneration after TBI (Faden and Loane, 2015; Simon et al., 2017).

The present study demonstrates that short-term depletion and subsequent repopulation of microglia during the chronic phase of experimental TBI reduces chronic neuroinflammation, improves neurological recovery (sensori-motor and cognitive function), and decreases neurodegeneration. These findings provide further evidence that the therapeutic window for targeting chronic neurodegeneration and improving functional recovery after TBI may be far longer than traditionally believed (i.e., hours post trauma) (Faden et al., 2016). One-week treatment with the CSF1R inhibitor, PLX5622, beginning at 1-month postinjury removed proinflammatory microglia from the chronically injured brain, and repopulated microglia had an altered phenotype that was markedly less inflammatory. Stereological assessments demonstrated that the repopulated microglia in the injured cortex displayed a more ramified morphology, similar to that of uninjured Sham controls. In contrast, Veh-treated TBI mice had cortical microglia with typical chronic posttraumatic hypertrophic morphology (Loane et al., 2014). Delayed short-term microglial depletion significantly improved neurological recovery through 3 months after injury, as demonstrated by a variety of complementary motor and cognitive function tests. Improved functional outcomes were associated with decreased cortical lesion volume and neu-
B
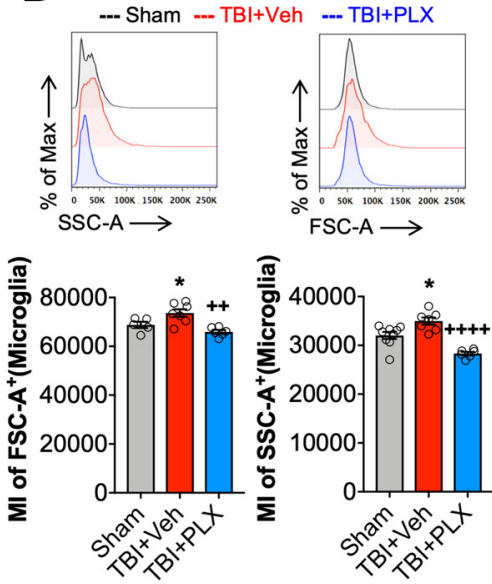

D
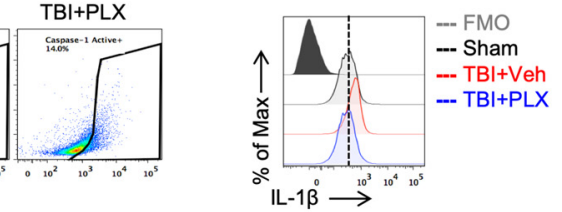

$\mathrm{IL}-1 \beta \longrightarrow$
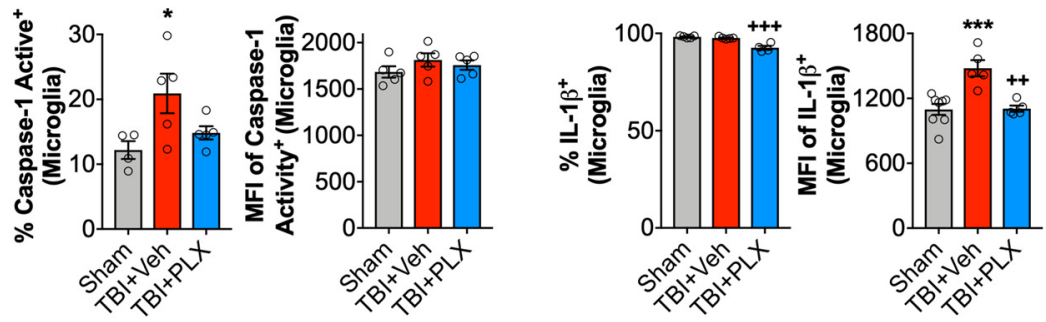

Figure 7. Delayed depletion of microglia during chronic TBI reduces expression of NLRP3 inflammasome components in microglia. A, Flow cytometry analysis and representative dot plots show populations of resident CD45 $5^{\text {int }}$ microglia and infiltrating peripherally derived CD45 ${ }^{\text {hi }}$ myeloid cells in the ipsilateral cortex at 2 months postinjury. There was no difference in absolute microglial numbers between Sham, TBI+Veh-treated, and TBI + PLX5622-treated groups. TBI increased numbers of infiltrating CD45 hi myeloid cells in brain in TBI + Veh-treated animals, but numbers were small compared with numbers of resident microglia. , Assessment of microglial cell size as measured by forward scatter (FSC), and microglial granularity as measured by side scatter (SSC), demonstrated that TBl increased microglial cell size and granularity when compared with levels in Sham microglia. PLX5622 treatment reduced both markers in microglia. C, Representative dot plots show resident CD45 ${ }^{\text {int }}$ microglia expressing active decreased by PLX5622 treatment, but failed to reach statistical significance. D, TBI increased IL-1 $\beta$ production in microglia, when compared with levels in Sham. Levels of microglial IL- $1 \beta$ was decreased in TBI + PLX5622-treated animals. ${ }^{*} p<0.05$, ${ }^{* * *} p<$ 0.001 , vs Sham; $++p<0.01,+++p<0.001,++++p=0.000$ vs TBI+Veh-treated animals. Data expressed as mean $\pm \operatorname{SEM}(n=5-9 /$ group).

ronal cell loss in the hippocampus. Thus, short-term depletion of microglia relatively late after TBI largely eliminates the destructive neurotoxic microglial phenotype that appears to contribute importantly to chronic posttraumatic neurodegeneration and associated neurological dysfunction.

Small-molecule CSF1R inhibitors have recently been used to investigate microglial dynamics under physiological and pathological conditions (Han et al., 2019). The latest generation CSF1R inhibitor, PLX5622, has desirable pharmacokinetic properties, including oral bioavailability with a $>20 \%$ brain penetrance, and it rapidly eliminates microglia ( $>95 \%$ ) in brain (Spangenberg et al., 2019). Despite microglia playing a crucial role in maintaining CNS homeostasis and synaptic plasticity (Salter and Stevens, 2017), depletion of microglia in adult mice using CSF1R inhibitors did not result in cognitive or motor function impairments 
A

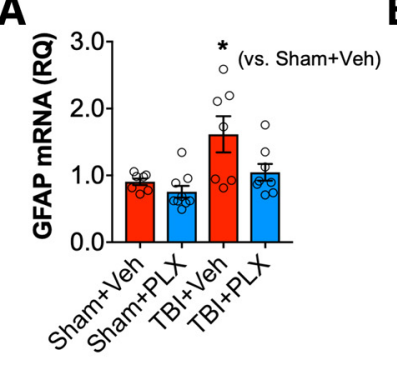

B ฐ

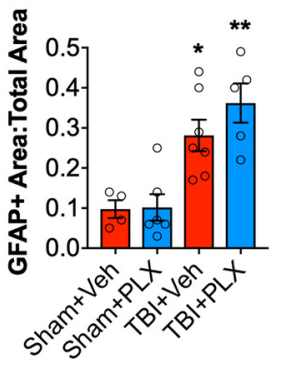

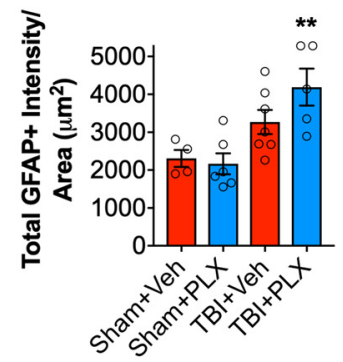

C
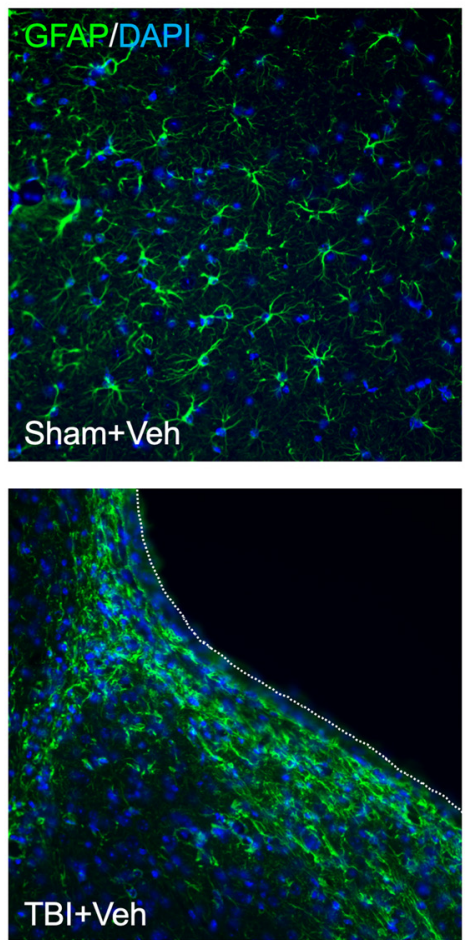
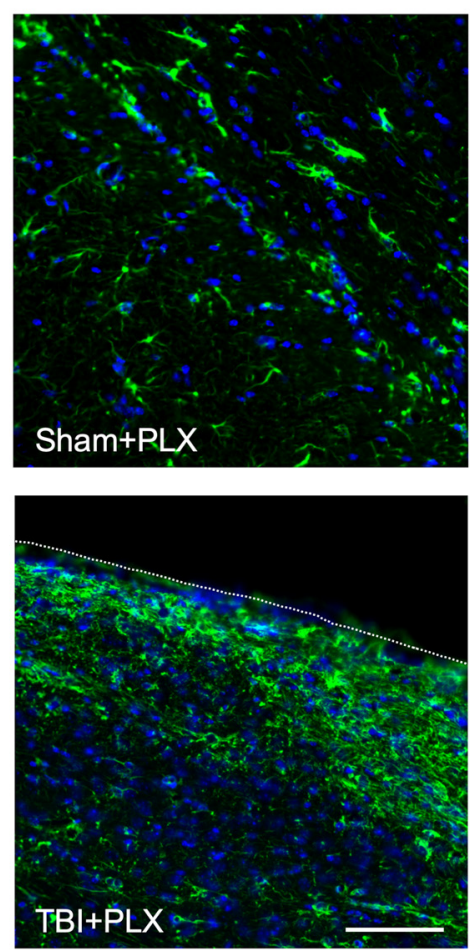

Figure 8. Delayed depletion of microglia during chronic TBI does not alter astrocyte reactivity in the cortex. $\boldsymbol{A}$, Analysis of ipsilateral cortical tissue at 2 months postinjury demonstrated that TBI increased Gfap mRNA. TBI-induced increase in Gfap mRNA was not altered by PLX5622 treatment. $\boldsymbol{B}$, In situ analysis of astrocytes in the perilesional cortex at 3 months postinjury. TBI increased astrocyte cellular area and GFAP expression when compared with levels in Sham. PLX5622 treatment did not change posttraumatic GFAP cellular distribution or expression. C, Representative images of GFAP (green) in the perilesional cortex at 3 months post injury. The dotted line represents the lesion boundary. Scale bar, $100 \mu \mathrm{m} .{ }^{*} p<0.05,{ }^{* *} p<0.01$ vs Sham. Data expressed as mean \pm SEM ( $n=5-8$ /group).

(Elmore et al., 2014). Similarly, we found that PLX5562 treatment of noninjured Sham control mice performed similar to Veh-treated Sham control (nondepleted) mice in complex cognitive, motor and affective behavioral tasks. Remarkably, short-term elimination of microglia late after TBI followed by repopulation markedly reduced impairments in sensori-motor function up to 3 months postinjury, exemplified by decreased numbers of foot faults on the BW task and reduced disability on a rotorod test. In addition, PLX5622-treated TBI mice had improved spatial and working memory, as shown by improved performance in the MWM, Y-maze, and NOR tests compared with Veh-treated TBI mice. The observed improvements in cognitive function recovery by the delayed PLX5622 treatment mimics long-term recovery trajectories mediated by a CSF1R inhibitor (PLX3397) in a hippocampal lesion model (Rice et al., 2015).

Previous work from our laboratory demonstrated that severe TBI induces chronic microglial activation up to one year postinjury and contributes to progressive lesion expansion, hippocam- pal neurodegeneration, and white matter damage (Loane et al., 2014). We reported that inhibiting chronic NOX2 activity after TBI suppressed microglial neurotoxicity, reduced chronic tissue loss and improved long-term motor and cognitive function recovery (Loane et al., 2013; Kumar et al., 2016a,b; Barrett et al., 2017). We also found that inhibition of chronic microglial activation beginning 1-month after TBI using either mGluR5 agonists or delayed aerobic exercise, reduced neurodegeneration and improved long-term neurological recovery (Byrnes et al., 2012; Piao et al., 2013). Both interventions significantly reduced NOX2 expression in chronically activated microglia. These, and other preclinical studies (Ferguson et al., 2017), provide additional evidence for an expanded therapeutic window for TBI by targeting chronic posttraumatic neuroinflammatory responses.

We used a Nanostring Neuropathology panel, which evaluates gene expression across fundamental pathways of neurodegeneration, to define networklevel changes in the injured cortex after chronic TBI. This whole tissue RNA analysis revealed that the neuroprotective effect of delayed PLX5622 treatment during chronic TBI were mediated by several beneficial mechanisms, including reconditioning the lesion microenvironment to be less proinflammatory, and reducing oxidative stress sensing and chronic NOX2 gene expression. Although our approach provides a global overview of gene expression changes in the injured cortex, it does not quantify the contribution of different cell types in the mixture to outcomes. Therefore, single cell studies will be required to identify cell-specific transcriptional programs in microglia that are modified by PLX5622 treatment, as well as changes that occur in other injured cells within the lesion microenvironment (i.e., neurons and astrocytes).

Given that activation of NLRP3 inflammasome has been linked to chronic neurodegeneration (Heneka et al., 2018) and TBI pathophysiology (Adamczak et al., 2012; Liu et al., 2013; Kerr et al., 2018; Xu et al., 2018; Kuwar et al., 2019), and that NOX2 may be an important upstream regulator of NLRP3 inflammasome activation (Dostert et al., 2008), we also examined molecular and cellular markers of this pathway. Key genes involved in NLRP3 inflammasome activation and function were chronically upregulated after TBI and reduced by PLX5622 treatment, including Illr1 and Casp1. Importantly, the inflammatory profile of repopulated microglia at 2 months postinjury showed decreased microglial IL- $1 \beta$ and caspase- 1 activation. Ma et al., recently reported robust activation of NLRP3 inflammasome in the injured cortex following experimental TBI, and that NOX2 inhibition markedly attenuated its activity (Ma et al., 2017). Whereas $\mathrm{Ma}$ et al., reported changes during the subacute phase after TBI 
(14 d postinjury (Ma et al., 2017)), we demonstrated chronic NLRP3 activation (active caspase- 1 and IL- $1 \beta$ expression) in microglia at 2 months postinjury. Thus, PLX5622 treatment confers neuroprotection after TBI, in part, by removing chronic NLRP3 inflammasome activation in microglia.

To assess the effect of depletion and subsequent microglial repopulation on other glial cells, we examined GFAP, a marker of astrocyte activation. Astrocyte reactivity depends on varying degrees of axonal injury, vascular disruption, ischemia and inflammation (Burda et al., 2016). Repopulated microglia did not significantly affect the TBI-induced increase in GFAP expression or reactivity (morphology) in perilesional cortex at 3 months postinjury. Although previous data regarding the effects of microglial depletion on acute GFAP changes after TBI are conflicting (Witcher et al., 2018), we did not observe significant changes in GFAP expression or morphology at chronic time points postTBI. Reported differences of PLX5622 treatment on posttraumatic astrocyte reactivity may reflect differences in the injury model (midline fluid percussion vs controlled cortical impact), time-points examined ( $3 \mathrm{~d}$ vs 3 months after injury), or the treatment paradigm used (pretreatment vs late posttrauma treatment). In addition, Witcher et al. reported that depletion of microglia before TBI did not reduce axonal injury in the cortex at $7 \mathrm{~d}$ postinjury (Witcher et al., 2018). In contrast, our study demonstrates that delayed depletion of microglia during the chronic stages of TBI significantly reduce neuronal cell loss in cortex and hippocampus. Again, such differences may reflect treatment timing or model.

Microglia depletion strategies are now regarded as promising translational therapies for neurological disorders (Han et al., 2019). Accumulating evidence indicates that microglial depletion has a broad range of neuroprotective effects in neurological diseases by reducing neuroinflammation. For example, in experimental models of Multiple Sclerosis microglial depletion reduces EAE progression (Nissen et al., 2018) and enhances remyelination in a cuprizone demyelination model (Beckmann et al., 2018). Furthermore, selective removal of microglia in AD models improves cognitive function, reduces neuronal loss, and partially prevents the progression of $\mathrm{AD}$ pathology, but has no effects on amyloid levels and plaque loads (Asai et al., 2015; Dagher et al., 2015; Olmos-Alonso et al., 2016; Spangenberg et al., 2016). However, microglial depletion has also been shown to exacerbate brain neurotoxicity in models of Parkinson's disease and coronavirus encephalitis (Wheeler et al., 2018; Yang et al., 2018). Further, microglial depletion in experimental stroke models exacerbates postischemic inflammation and acute brain injury (Szalay et al., 2016; Jin et al., 2017). Therefore, further research is needed to identify the mechanisms by which microglial depletion confers neuroprotection before targeted microglial depletion strategies are translated to the clinic for neurological diseases. Another significant issue is that small-molecule CSF1R inhibitors (including PLX5562) might have off-target effects and deplete cell populations other than microglia. Other tissue resident macrophages express CSF1R (Hume et al., 2019), suggesting that PLX5622 treatment may also affect peripheral immune responses. Therefore, cautious interpretation of pure microglial depletion effects using systemic delivery of currently available small-molecule CSF1R inhibitors is warranted.

Together, our preclinical studies strongly support the concept that chronic microglial activation contributes to posttraumatic neurodegeneration and related neurological dysfunction after TBI. The neuroprotective effects of short-term depletion of chronically activated microglia late after TBI likely reflect multi- factorial mechanisms- including less NOX2-mediated neurotoxic inflammation associated with altered oxidative stress responses, and reduced expression of the NLRP3 inflammasome in reactive microglia and release of proinflammatory IL-1 $\beta$. These studies support new concepts that the therapeutic window for TBI may be far longer than traditionally believed if chronic and evolving neuroinflammation can be inhibited or regulated in a precise manner (Faden and Loane, 2015). Furthermore, they suggest that additional investigation of CSF1R inhibitors as a clinically feasible approach to limit posttraumatic neurodegeneration and neurological dysfunction following TBI is required.

\section{References}

Adamczak S, Dale G, de Rivero Vaccari JP, Bullock MR, Dietrich WD, Keane RW (2012) Inflammasome proteins in cerebrospinal fluid of braininjured patients as biomarkers of functional outcome: clinical article. J Neurosurg 117:1119-1125.

Asai H, Ikezu S, Tsunoda S, Medalla M, Luebke J, Haydar T, Wolozin B, Butovsky O, Kügler S, Ikezu T (2015) Depletion of microglia and inhibition of exosome synthesis halt tau propagation. Nat Neurosci 18:1584-1593.

Babior BM (1999) NADPH oxidase: an update. Blood 93:1464-1476.

Barrett JP, Henry RJ, Villapol S, Stoica BA, Kumar A, Burns MP, Faden AI, Loane DJ (2017) NOX2 deficiency alters macrophage phenotype through an IL-10/STAT3 dependent mechanism: implications for traumatic brain injury. J Neuroinflammation 14:65.

Beckmann N, Giorgetti E, Neuhaus A, Zurbruegg S, Accart N, Smith P, Perdoux J, Perrot L, Nash M, Desrayaud S, Wipfli P, Frieauff W, Shimshek DR (2018) Brain region-specific enhancement of remyelination and prevention of demyelination by the CSF1R kinase inhibitor BLZ945. Acta Neuropathol Commun 6:9.

Bendlin BB, Ries ML, Lazar M, Alexander AL, Dempsey RJ, Rowley HA, Sherman JE, Johnson SC (2008) Longitudinal changes in patients with traumatic brain injury assessed with diffusion-tensor and volumetric imaging. Neuroimage 42:503-514.

Block ML, Zecca L, Hong JS (2007) Microglia-mediated neurotoxicity: uncovering the molecular mechanisms. Nat Rev Neurosci 8:57-69.

Brody DL, Holtzman DM (2006) Morris water maze search strategy analysis in PDAPP mice before and after experimental traumatic brain injury. Exp Neurol 197:330-340.

Bryant RA, O’Donnell ML, Creamer M, McFarlane AC, Clark CR, Silove D (2010) The psychiatric sequelae of traumatic injury. Am J Psychiatry 167:312-320.

Burda JE, Bernstein AM, Sofroniew MV (2016) Astrocyte roles in traumatic brain injury. Exp Neurol 275:305-315.

Byrnes KR, Loane DJ, Stoica BA, Zhang J, Faden AI (2012) Delayed mGluR5 activation limits neuroinflammation and neurodegeneration after traumatic brain injury. J Neuroinflammation 9:43.

Cherry JD, Tripodis Y, Alvarez VE, Huber B, Kiernan PT, Daneshvar DH, Mez J, Montenigro PH, Solomon TM, Alosco ML, Stern RA, McKee AC, Stein TD (2016) Microglial neuroinflammation contributes to tau accumulation in chronic traumatic encephalopathy. Acta Neuropathol Commun 4:112.

Coughlin JM, Wang Y, Minn I, Bienko N, Ambinder EB, Xu X, Peters ME, Dougherty JW, Vranesic M, Koo SM, Ahn HH, Lee M, Cottrell C, Sair HI, Sawa A, Munro CA, Nowinski CJ, Dannals RF, Lyketsos CG, Kassiou M, Smith G, et al. (2017) Imaging of glial cell activation and white matter integrity in brains of active and recently retired national football league players. JAMA Neurol 74:67-74.

Coughlin JM, Wang Y, Munro CA, Ma S, Yue C, Chen S, Airan R, Kim PK, Adams AV, Garcia C, Higgs C, Sair HI, Sawa A, Smith G, Lyketsos CG, Caffo B, Kassiou M, Guilarte TR, Pomper MG (2015) Neuroinflammation and brain atrophy in former NFL players: an in vivo multimodal imaging pilot study. Neurobiol Dis 74:58-65.

Dagher NN, Najafi AR, Kayala KM, Elmore MR, White TE, Medeiros R, West BL, Green KN (2015) Colony-stimulating factor 1 receptor inhibition prevents microglial plaque association and improves cognition in 3xTg-AD mice. J Neuroinflammation 12:139.

Dams-O'Connor K, Gibbons LE, Bowen JD, McCurry SM, Larson EB, Crane PK (2013) Risk for late-life re-injury, dementia and death among indi- 
viduals with traumatic brain injury: a population-based study. J Neurol Neurosurg Psychiatry 84:177-182.

Doran SJ, Ritzel RM, Glaser EP, Henry RJ, Faden AI, Loane DJ (2019) Sex differences in acute neuroinflammation after experimental traumatic brain injury are mediated by infiltrating myeloid cells. J Neurotrauma 36:1040-1053.

Dostert C, Pétrilli V, Van Bruggen R, Steele C, Mossman BT, Tschopp J (2008) Innate immune activation through Nalp3 inflammasome sensing of asbestos and silica. Science 320:674-677.

Elmore MR, Najafi AR, Koike MA, Dagher NN, Spangenberg EE, Rice RA, Kitazawa M, Matusow B, Nguyen H, West BL, Green KN (2014) Colony-stimulating factor 1 receptor signaling is necessary for microglia viability, unmasking a microglia progenitor cell in the adult brain. Neuron 82:380-397.

Elmore MR, Lee RJ, West BL, Green KN (2015) Characterizing newly repopulated microglia in the adult mouse: impacts on animal behavior, cell morphology, and neuroinflammation. PLoS One 10:e0122912.

Erblich B, Zhu L, Etgen AM, Dobrenis K, Pollard JW (2011) Absence of colony stimulation factor-1 receptor results in loss of microglia, disrupted brain development and olfactory deficits. PLoS One 6:e26317.

Faden AI, Loane DJ (2015) Chronic neurodegeneration after traumatic brain injury: alzheimer disease, chronic traumatic encephalopathy, or persistent neuroinflammation? Neurotherapeutics 12:143-150.

Faden AI, Wu J, Stoica BA, Loane DJ (2016) Progressive inflammationmediated neurodegeneration after traumatic brain or spinal cord injury. Br J Pharmacol 173:681-691.

Ferguson S, Mouzon B, Paris D, Aponte D, Abdullah L, Stewart W, Mullan M, Crawford F (2017) Acute or delayed treatment with anatabine improves spatial memory and reduces pathological sequelae at late time-points after repetitive mild traumatic brain injury. J Neurotrauma 34:1676-1691.

Foucher Y, Danger R (2012) Time dependent ROC curves for the estimation of true prognostic capacity of microarray data. Stat Appl Genet Mol Biol 11:Article 1.

García-Cabezas MÁ, John YJ, Barbas H, Zikopoulos B (2016) Distinction of neurons, glia and endothelial cells in the cerebral cortex: an algorithm based on cytological features. Front Neuroanat 10:107.

Gardner RC, Burke JF, Nettiksimmons J, Kaup A, Barnes DE, Yaffe K (2014) Dementia risk after traumatic brain injury vs nonbrain trauma: the role of age and severity. JAMA Neurol 71:1490-1497.

Gentleman SM, Leclercq PD, Moyes L, Graham DI, Smith C, Griffin WS, Nicoll JA (2004) Long-term intracerebral inflammatory response after traumatic brain injury. Forensic Sci Int 146:97-104.

Ginhoux F, Greter M, Leboeuf M, Nandi S, See P, Gokhan S, Mehler MF, Conway SJ, Ng LG, Stanley ER, Samokhvalov IM, Merad M (2010) Fate mapping analysis reveals that adult microglia derive from primitive macrophages. Science 330:841-845.

Han J, Zhu K, Zhang XM, Harris RA (2019) Enforced microglial depletion and repopulation as a promising strategy for the treatment of neurological disorders. Glia 67:217-231.

Heneka MT, Carson MJ, El Khoury J, Landreth GE, Brosseron F, Feinstein DL, Jacobs AH, Wyss-Coray T, Vitorica J, Ransohoff RM, Herrup K, Frautschy SA, Finsen B, Brown GC, Verkhratsky A, Yamanaka K, Koistinaho J, Latz E, Halle A, Petzold GC et al. (2015) Neuroinflammation in Alzheimer's disease. Lancet Neurol 14:388-405.

Heneka MT, McManus RM, Latz E (2018) Inflammasome signalling in brain function and neurodegenerative disease. Nat Rev Neurosci 19: $610-621$.

Henry RJ, Doran SJ, Barrett JP, Meadows VE, Sabirzhanov B, Stoica BA, Loane DJ, Faden AI (2019) Inhibition of miR-155 limits neuroinflammation and improves functional recovery after experimental traumatic brain injury in mice. Neurotherapeutics 16:216-230.

Hume DA, Irvine KM, Pridans C (2019) The mononuclear phagocyte system: the relationship between monocytes and macrophages. Trends Immunol 40:98-112.

Jin WN, Shi SX, Li Z, Li M, Wood K, Gonzales RJ, Liu Q (2017) Depletion of microglia exacerbates postischemic inflammation and brain injury. J Cereb Blood Flow Metab 37:2224-2236.

Johnson VE, Stewart JE, Begbie FD, Trojanowski JQ, Smith DH, Stewart W (2013) Inflammation and white matter degeneration persist for years after a single traumatic brain injury. Brain 136:28-42.

Kerr N, Lee SW, Perez-Barcena J, Crespi C, Ibañez J, Bullock MR, Dietrich
WD, Keane RW, de Rivero Vaccari JP (2018) Inflammasome proteins as biomarkers of traumatic brain injury. PLoS One 13:e0210128.

Kumar A, Alvarez-Croda DM, Stoica BA, Faden AI, Loane DJ (2016a) Microglial/macrophage polarization dynamics following traumatic brain injury. J Neurotrauma 33:1732-1750.

Kumar A, Barrett JP, Alvarez-Croda DM, Stoica BA, Faden AI, Loane DJ (2016b) NOX2 drives M1-like microglial/macrophage activation and neurodegeneration following experimental traumatic brain injury. Brain Behav Immun 58:291-309.

Kumar RG, Gao S, Juengst SB, Wagner AK, Fabio A (2018) The effects of post-traumatic depression on cognition, pain, fatigue, and headache after moderate-to-severe traumatic brain injury: a thematic review. Brain Inj 32:383-394.

Kuwar R, Rolfe A, Di L, Xu H, He L, Jiang Y, Zhang S, Sun D (2019) A novel small molecular NLRP3 inflammasome inhibitor alleviates neuroinflammatory response following traumatic brain injury. J Neuroinflammation $16: 81$.

Lage SL, Dominical VM, Wong CS, Sereti I (2019) Evaluation of canonical inflammasome activation in human monocytes by imaging flow cytometry. Front Immunol 10:1284.

Li M, Li Z, Ren H, Jin WN, Wood K, Liu Q, Sheth KN, Shi FD (2017) Colony stimulating factor 1 receptor inhibition eliminates microglia and attenuates brain injury after intracerebral hemorrhage. J Cereb Blood Flow Metab 37:2383-2395.

Liddelow SA, Guttenplan KA, Clarke LE, Bennett FC, Bohlen CJ, Schirmer L, Bennett ML, Münch AE, Chung WS, Peterson TC, Wilton DK, Frouin A, Napier BA, Panicker N, Kumar M, Buckwalter MS, Rowitch DH, Dawson VL, Dawson TM, Stevens B, et al. (2017) Neurotoxic reactive astrocytes are induced by activated microglia. Nature 541:481-487.

Liu HD, Li W, Chen ZR, Hu YC, Zhang DD, Shen W, Zhou ML, Zhu L, Hang $\mathrm{CH}$ (2013) Expression of the NLRP3 inflammasome in cerebral cortex after traumatic brain injury in a rat model. Neurochem Res 38:20722083.

Loane DJ, Faden AI (2010) Neuroprotection for traumatic brain injury: translational challenges and emerging therapeutic strategies. Trends Pharmacol Sci 31:596-604.

Loane DJ, Kumar A (2016) Microglia in the TBI brain: the good, the bad, and the dysregulated. Exp Neurol 275:316-327.

Loane DJ, Stoica BA, Byrnes KR, Jeong W, Faden AI (2013) Activation of mGluR5 and inhibition of NADPH oxidase improves functional recovery after traumatic brain injury. J Neurotrauma 30:403-412.

Loane DJ, Kumar A, Stoica BA, Cabatbat R, Faden AI (2014) Progressive neurodegeneration after experimental brain trauma: association with chronic microglial activation. J Neuropathol Exp Neurol 73:14-29.

Ma MW, Wang J, Dhandapani KM, Brann DW (2017) NADPH oxidase 2 regulates NLRP3 inflammasome activation in the brain after traumatic brain injury. Oxid Med Cell Longev 2017:6057609.

Maas AIR, Menon DK, Adelson PD, Andelic N, Bell MJ, Belli A, Bragge P, Brazinova A, Büki A, Chesnut RM, Citerio G, Coburn M, Cooper DJ, Crowder AT, Czeiter E, Czosnyka M, Diaz-Arrastia R, Dreier JP, Duhaime AC, Ercole A, et al. (2017) Traumatic brain injury: integrated approaches to improve prevention, clinical care, and research. Lancet Neurol 16:987-1048.

Maxwell WL, MacKinnon MA, Smith DH, McIntosh TK, Graham DI (2006) Thalamic nuclei after human blunt head injury. J Neuropathol Exp Neurol 65:478-488.

Mortimer JA, French LR, Hutton JT, Schuman LM (1985) Head injury as a risk factor for Alzheimer's disease. Neurology 35:264-267.

Nandi S, Gokhan S, Dai XM, Wei S, Enikolopov G, Lin H, Mehler MF, Stanley ER (2012) The CSF-1 receptor ligands IL-34 and CSF-1 exhibit distinct developmental brain expression patterns and regulate neural progenitor cell maintenance and maturation. Dev Biol 367:100-113.

Nissen JC, Thompson KK, West BL, Tsirka SE (2018) Csf1R inhibition attenuates experimental autoimmune encephalomyelitis and promotes recovery. Exp Neurol 307:24-36.

Nordström P, Michaëlsson K, Gustafson Y, Nordström A (2014) Traumatic brain injury and young onset dementia: a nationwide cohort study. Ann Neurol 75:374-381.

Olmos-Alonso A, Schetters ST, Sri S, Askew K, Mancuso R, Vargas-Caballero M, Holscher C, Perry VH, Gomez-Nicola D (2016) Pharmacological targeting of CSF1R inhibits microglial proliferation and prevents the progression of Alzheimer's-like pathology. Brain 139:891-907. 
Perry VH, Nicoll JA, Holmes C (2010) Microglia in neurodegenerative disease. Nat Rev Neurol 6:193-201.

Piao CS, Stoica BA, Wu J, Sabirzhanov B, Zhao Z, Cabatbat R, Loane DJ, Faden AI (2013) Late exercise reduces neuroinflammation and cognitive dysfunction after traumatic brain injury. Neurobiol Dis 54:252-263.

Pischiutta F, Micotti E, Hay JR, Marongiu I, Sammali E, Tolomeo D, Vegliante G, Stocchetti N, Forloni G, De Simoni MG, Stewart W, Zanier ER (2018) Single severe traumatic brain injury produces progressive pathology with ongoing contralateral white matter damage one year after injury. Exp Neurol 300:167-178.

Qin L, Liu Y, Wang T, Wei SJ, Block ML, Wilson B, Liu B, Hong JS (2004) NADPH oxidase mediates lipopolysaccharide-induced neurotoxicity and proinflammatory gene expression in activated microglia. J Biol Chem 279:1415-1421.

Ramlackhansingh AF, Brooks DJ, Greenwood RJ, Bose SK, Turkheimer FE, Kinnunen KM, Gentleman S, Heckemann RA, Gunanayagam K, Gelosa G, Sharp DJ (2011) Inflammation after trauma: microglial activation and traumatic brain injury. Ann Neurol 70:374-383.

Rice RA, Spangenberg EE, Yamate-Morgan H, Lee RJ, Arora RP, Hernandez MX, Tenner AJ, West BL, Green KN (2015) Elimination of microglia improves functional outcomes following extensive neuronal loss in the hippocampus. J Neurosci 35:9977-9989.

Rice RA, Pham J, Lee RJ, Najafi AR, West BL, Green KN (2017) Microglial repopulation resolves inflammation and promotes brain recovery after injury. Glia 65:931-944.

Ritzel RM, Doran SJ, Barrett JP, Henry RJ, Ma EL, Faden AI, Loane DJ (2018) Chronic alterations in systemic immune function after traumatic brain injury. J Neurotrauma 35:1419-1436.

Ritzel RM, Doran SJ, Glaser EP, Meadows VE, Faden AI, Stoica BA, Loane DJ (2019) Old age increases microglial senescence, exacerbates secondary neuroinflammation, and worsens neurological outcomes after acute traumatic brain injury in mice. Neurobiol Aging 77:194-206.

Rohart F, Gautier B, Singh A, Lê Cao KA (2017) mixOmics: An R package for 'omics feature selection and multiple data integration. PLoS Comput Biol 13:e1005752.

Sabaz M, Simpson GK, Walker AJ, Rogers JM, Gillis I, Strettles B (2014) Prevalence, comorbidities, and correlates of challenging behavior among community-dwelling adults with severe traumatic brain injury: a multicenter study. J Head Trauma Rehabil 29:E19-E30.

Salter MW, Stevens B (2017) Microglia emerge as central players in brain disease. Nat Med 23:1018-1027.

Simon DW, McGeachy MJ, Bayır H, Clark RS, Loane DJ, Kochanek PM (2017) The far-reaching scope of neuroinflammation after traumatic brain injury. Nat Rev Neurol 13:171-191.
Spangenberg E, Severson PL, Hohsfield LA, Crapser J, Zhang J, Burton EA, Zhang Y, Spevak W, Lin J, Phan NY, Habets G, Rymar A, Tsang G, Walters J, Nespi M, Singh P, Broome S, Ibrahim P, Zhang C, Bollag G, West BL, et al. (2019) Sustained microglial depletion with CSF1R inhibitor impairs parenchymal plaque development in an Alzheimer's disease model. Nat Commun 10:3758.

Spangenberg EE, Lee RJ, Najafi AR, Rice RA, Elmore MR, Blurton-Jones M, West BL, Green KN (2016) Eliminating microglia in Alzheimer's mice prevents neuronal loss without modulating amyloid-beta pathology. Brain 139:1265-1281.

Szalay G, Martinecz B, Lénárt N, Környei Z, Orsolits B, Judák L, Császár E, Fekete R, West BL, Katona G, Rózsa B, Dénes Á (2016) Microglia protect against brain injury and their selective elimination dysregulates neuronal network activity after stroke. Nat Commun 7:11499.

Villacampa N, Almolda B, Vilella A, Campbell IL, González B, Castellano B (2015) Astrocyte-targeted production of IL-10 induces changes in microglial reactivity and reduces motor neuron death after facial nerve axotomy. Glia 63:1166-1184.

Wang H, Horbinski C, Wu H, Liu Y, Sheng S, Liu J, Weiss H, Stromberg AJ, Wang C (2016) NanoStringDiff: a novel statistical method for differential expression analysis based on NanoString nCounter data. Nucleic Acids Res 44:e151.

Wheeler DL, Sariol A, Meyerholz DK, Perlman S (2018) Microglia are required for protection against lethal coronavirus encephalitis in mice. J Clin Invest 128:931-943.

Wilson L, Stewart W, Dams-O'Connor K, Diaz-Arrastia R, Horton L, Menon DK, Polinder S (2017) The chronic and evolving neurological consequences of traumatic brain injury. Lancet Neurol 16:813-825.

Witcher KG, Bray CE, Dziabis JE, McKim DB, Benner BN, Rowe RK, KokikoCochran ON, Popovich PG, Lifshitz J, Eiferman DS, Godbout JP (2018) Traumatic brain injury-induced neuronal damage in the somatosensory cortex causes formation of rod-shaped microglia that promote astrogliosis and persistent neuroinflammation. Glia 66:2719-2736.

Xu X, Yin D, Ren H, Gao W, Li F, Sun D, Wu Y, Zhou S, Lyu L, Yang M, Xiong J, Han L, Jiang R, Zhang J (2018) Selective NLRP3 inflammasome inhibitor reduces neuroinflammation and improves long-term neurological outcomes in a murine model of traumatic brain injury. Neurobiol Dis 117:15-27.

Yang X, Ren H, Wood K, Li M, Qiu S, Shi FD, Ma C, Liu Q (2018) Depletion of microglia augments the dopaminergic neurotoxicity of MPTP. FASEB J 32:3336-3345.

Zhao Z, Loane DJ, Murray MG 2nd, Stoica BA, Faden AI (2012) Comparing the predictive value of multiple cognitive, affective, and motor tasks after rodent traumatic brain injury. J Neurotrauma 29:2475-2489. 